Menglan Liao, Qiang Liu, and Hailong Ye*

\title{
Global existence and blow-up of weak solutions for a class of fractional $p$-Laplacian evolution equations
}

https://doi.org/10.1515/anona-2020-0066

Received March 22, 2019; accepted February 20, 2020.

Abstract: In this paper, we study the fractional $p$-Laplacian evolution equation with arbitrary initial energy,

$$
u_{t}(x, t)+(-\Delta)_{p}^{s} u(x, t)=f(u(x, t)), \quad x \in \Omega, t>0,
$$

where $(-\Delta)_{p}^{s}$ is the fractional $p$-Laplacian with $p>\max \left\{\frac{2 N}{N+2 s}, 1\right\}$ and $s \in(0,1)$. Specifically, by the modified potential well method, we obtain the global existence, uniqueness, and blow-up in finite time of the weak solution for the low, critical and high initial energy cases respectively.

Keywords: fractional $p$-Laplacian evolution equation; potential well; global existence; uniqueness; blow-up

MSC 2010: 35R11; 35K20; 35B44; 35D30

\section{Introduction}

Let $\Omega \subset \mathbb{R}^{N}(N \geqslant 1)$ be a bounded domain with smooth boundary, $T \in(0, \infty], p>\max \left\{\frac{2 N}{N+2 S}, 1\right\}$ and $s \in(0,1)$. In this paper, we study the following fractional $p$-Laplacian evolution equation with the zero Dirichlet boundary value condition:

$$
\begin{cases}u_{t}(x, t)+(-\Delta)_{p}^{s} u(x, t)=f(u(x, t)) & (x, t) \in \Omega \times(0, T), \\ u(x, t)=0 & (x, t) \in\left(\mathbb{R}^{N} \backslash \Omega\right) \times(0, T), \\ u(x, 0)=u_{0}(x) & x \in \Omega,\end{cases}
$$

where the non-local fractional $p$-Laplacian operator $(-\Delta)_{p}^{s} u$ is defined as

$$
(-\Delta)_{p}^{S} u(x)=\lim _{\varepsilon \downarrow 0} \int_{\mathbb{R}^{N} \backslash B_{\varepsilon}(x)} \frac{|u(x)-u(y)|^{p-2}(u(x)-u(y))}{|x-y|^{N+p s}} \mathrm{~d} y .
$$

Here, the function $f$ is a given function satisfying the following conditions:

(a) $f \in C^{1}$ and $f(0)=f^{\prime}(0)=0$.

(b) $f(u)$ is monotone, convex for $u>0$ and concave for $u<0$.

(c) $(q+1) F(u) \leqslant u f(u)$ and $|u f(u)| \leqslant r|F(u)|$, where $F(u)=\int_{0}^{u} f(s) d s$ and

$$
\max \left\{\frac{2 N}{N+2 s}, 1\right\}<p, \quad \max \{p, 2\}<q+1 \leqslant r<p_{s}^{\star}
$$

\footnotetext{
Menglan Liao, School of Mathematics, Jilin University, Changchun, Jilin Province 130012, China Department of Mathematics, Michigan State University, East Lansing, MI 48824, USA

Qiang Liu, College of Mathematics and Statistics, Shenzhen University, Shenzhen, 518060, China

*Corresponding Author: Hailong Ye, College of Mathematics and Statistics, Shenzhen University, Shenzhen, 518060, China Shenzhen Key Laboratory of Advanced Machine Learing and Applications, Shenzhen University, Shenzhen, 518060, China, E-mail:ye2006hailong@yeah.net
} 
where

$$
p_{s}^{\star}= \begin{cases}\frac{N p}{N-s p} & \text { if } s p<N, \\ +\infty & \text { if } s p \geq N .\end{cases}
$$

(d) $u\left(u f^{\prime}(u)-(p-1) f(u)\right) \geqslant 0$ and the equality holds only for $u=0$.

For instance, we could take $f(u)=\lambda_{1}|u|^{r-2} u+\lambda_{2}|u|^{q-1} u$ with $\lambda_{1}, \lambda_{2} \geqslant 0$.

The fractional Laplacian as a generalization of the integer order Laplace operator has been studied in classical monographs such as $[14,30]$ and so on. Recently, more attention has been focused on the study of the differential equation involved with non-local fractional operators. These equations could be used in many fields, such as digit image processing [10], obstacle problem [28], phase transitions [2] and so on. We also refer the reader to the monographs $[3,6,20]$ and the references therein for a fairly large description of rigorous mathematics and diverse applications. Usually, non-local generalizations of the Laplacian (both linear and nonlinear) are examined with smooth kernels and the fractional Laplacian is represented as an integral operator over the whole $\mathbb{R}^{N}$. In this work, we focus on non-local operators acting on bounded domains which correspond to the regional fractional Laplacian and can be interpreted as a nonlocal version of the Laplacian equipped with Dirichlet boundary conditions (see [24]). The non-local generalization of the $p$-Laplacian we are going to study is hence the nonlinear pendant to the fractional regional Laplacian mentioned above and appears, for instance, as a type of nonlinear diffusion [31]. Here, we mention that though it is also possible to define the fractional Laplacian via Fourier transform, this approach is restricted to the case of $p=2$.

In 2016, Vázquez [32] considered the existence, uniqueness and a number of quantitative properties of strong nonnegative solutions of a Dirichlet problem with fractional $p$-Laplacian evolution equation

$$
u_{t}(x, t)+(-\Delta)_{p}^{s} u(x, t)=0,
$$

where $0<s<1$ and $p>1$. When equation (1.3) is coupled with the Neumann boundary condition and the Cauchy initial condition, the existence, uniqueness and asymptotic behavior of strong solutions are obtained by the semigroup methods in [19]. Very recently, authors in [1] established the existence and suitable regularity of the weak solution and the entropy solution with general data. They also gave some properties of the solution, such as extinction, nonfinite speed of propagation, according to the values of $p$. Some regularities are also established in [12].

When $p=2$ and $f$ is nonlinear, Musso et al. [21] consider infinite time blow-up for positive solutions of the fractional heat equation with critical exponent

$$
\begin{cases}u_{t}=-(-\Delta)^{s} u+u^{\frac{N+2 s}{N-2 s}} & \text { in } \Omega \times(0, \infty), \\ u=0 & \text { on }\left(\mathbb{R}^{N} \backslash \Omega\right) \times(0, \infty), \\ u(\cdot, 0)=u_{0} & \text { in } \mathbb{R}^{N},\end{cases}
$$

where $N>4 s$ and $s \in(0,1)$. Later, the finite time blow-up problem for (1.4) with $4 s<N<6 s$ was investigated in [4]. When $p \neq 2$, Gal and Warm [8] studied the existence of the strong solution and the blow-up for the initial data with finite energy and a positive low bound. For the bounded initial data, the existence, uniqueness and global behavior of solutions are proved in [9]. Recently, Pan, Zhang and Cao [22] studied the following parabolic equation involving the fractional $p$-Laplacian:

$$
\begin{cases}u_{t}(x, t)+[u]_{s, p}^{(\lambda-1) p}(-\Delta)_{p}^{s} u=|u|^{q-2} u & (x, t) \in \Omega \times(0, T), \\ u(x, t)=0 & (x, t) \in\left(\mathbb{R}^{N} \backslash \Omega\right) \times(0, T), \\ u(x, 0)=u_{0}(x) & x \in \Omega,\end{cases}
$$

where $[u]_{s, p}$ is the Gagliardo $p$-seminorm of $u, p<q<\frac{N p}{N-s p}$ with $1<p<\frac{N}{s}$ and $1 \leqslant \lambda<\frac{N}{N-s p}$. Under some appropriate assumptions, the authors obtained the existence of a global solution to the problem (1.5) by the Galerkin method and potential well theory under $J\left(u_{0}\right)=d$ or $0<J\left(u_{0}\right)<d$, where $J\left(u_{0}\right)$ is the initial 
energy. However, authors did not study the problem for $J\left(u_{0}\right)>d$ and blow-up in finite time for arbitrary initial energy. In this paper, we consider the global existence and blow-up in finite time for the problem (1.1) for the more general $f(u)$ when the initial energy $J\left(u_{0}\right)$ is arbitrary.

Potential well method was firstly proposed by Sattinger [25] to study non-linear hyperbolic boundaryinitial value problem. Since then, many authors have studied the existence of solutions for evolution equations by potential well theory $[17,18,23,34]$. Especially, authors $[17,18,34]$ improved the results of Sattinger's by introducing a family of potential wells not only obtained some new results on global existence and invariant sets of solutions, but also discovered the vacuum isolating of solutions. Recently, the properties of a class of fourth-order parabolic equation were obtained with $J\left(u_{0}\right)>d$ in $[7,11,35]$.

In this paper, motivated by the works mentioned above, we consider the global existence, uniqueness and blow-up property of problem (1.1) with arbitrary initial energy $J\left(u_{0}\right)$. The main results regarding the low initial energy $\left(J\left(u_{0}\right)<d\right)$ are as following. The notations used below are defined in Section 2 and 3.

Theorem 1.1. Let conditions $(a)-(d)$ hold and $u_{0} \in X_{0}$. If $J\left(u_{0}\right)<d$ and $I\left(u_{0}\right)>0$, then problem (1.1) admits a global weak solution $u \in L^{\infty}\left(0, \infty ; X_{0}\right)$ with $u_{t} \in L^{2}\left(0, \infty ; L^{2}(\Omega)\right)$ and $u(t) \in W$ for $0 \leqslant t<\infty$. The weak solution is unique if it is bounded. Moreover, there exists $\delta_{1} \in(0,1)$ such that the following estimates hold:

(i) if $p<2$, then

$$
\|u\|_{L^{2}(\Omega)}^{2} \leqslant\left(\left\|u_{0}\right\|_{L^{2}(\Omega)}^{2-p}-(2-p)\left(1-\delta_{1}\right) C_{\star}^{-p} t\right)_{+}^{\frac{2}{2-p}}, \quad \forall t \geqslant 0,
$$

where $(x)_{+}:=\max \{x, 0\}$, which implies $u$ vanishes in finite time;

(ii) if $p=2$, then

$$
\|u(t)\|_{L^{2}(\Omega)}^{2} \leqslant\left\|u_{0}\right\|_{L^{2}(\Omega)}^{2} e^{-2\left(1-\delta_{1}\right) C_{*}^{-2} t}, \quad \forall t \geqslant 0
$$

(iii) if $p>2$, then

$$
\|u(t)\|_{L^{2}(\Omega)}^{2} \leqslant\left(\frac{1}{(p-2)\left(1-\delta_{1}\right) C_{\star}^{-p} t+\left\|u_{0}\right\|_{L^{2}(\Omega)}^{2-p}}\right)^{\frac{2}{p-2}}, \quad \forall t \geqslant 0 .
$$

Theorem 1.2. Let conditions $(a)-(d)$ hold and $u_{0} \in X_{0}$. If $J\left(u_{0}\right)<d$ and $I\left(u_{0}\right)<0$, then there exists a finite time $T^{\star}$ such that the weak solution of problem (1.1) blows up in the sense of

$$
\lim _{t \rightarrow T^{\star}} \int_{0}^{t}\|u\|_{L^{2}(\Omega)}^{2} \mathrm{~d} \tau=+\infty
$$

Next, we are concerned with the case of the critical initial energy $\left(J\left(u_{0}\right)=d\right)$.

Theorem 1.3. Let conditions $(a)-(d)$ hold and $u_{0} \in X_{0}$. If $J\left(u_{0}\right)=d$ and $I\left(u_{0}\right) \geqslant 0$, then problem (1.1) admits a global weak solution $u \in L^{\infty}\left(0, \infty ; X_{0}\right)$ with $u_{t} \in L^{2}\left(0, \infty ; L^{2}(\Omega)\right)$ and $u(t) \in W \cup \partial W$ for $0 \leqslant t<\infty$. The weak solution is unique if it is bounded. If $I(u)>0$ for $0<t<\infty$, then there exists $t_{0}>0$ and $\delta_{1} \in(0,1)$ such that the following estimates hold:

(i) if $p<2$, then

$$
\|u\|_{L^{2}(\Omega)}^{2} \leqslant\left(\left\|u\left(t_{0}\right)\right\|_{L^{2}(\Omega)}^{2-p}-(2-p)\left(1-\delta_{1}\right) C_{*}^{-p} t\right)_{+}^{\frac{2}{2-p}}, \quad \forall t \geqslant t_{0},
$$

which implies $u$ vanishes in finite time;

(ii) if $p=2$, then

$$
\|u(t)\|_{L^{2}(\Omega)}^{2} \leqslant\left\|u_{0}\right\|_{L^{2}(\Omega)}^{2} e^{-2\left(1-\delta_{1}\right) C_{*}^{-2} t}, \quad \forall t \geqslant 0
$$

(iii) if $p>2$, then

$$
\|u(t)\|_{L^{2}(\Omega)}^{2} \leqslant\left(\frac{1}{(p-2)\left(1-\delta_{1}\right) C_{\star}^{-p} t+\left\|u_{0}\right\|_{L^{2}(\Omega)}^{2-p}}\right)^{\frac{2}{p-2}}, \quad \forall t \geqslant 0 .
$$


If there exists $t^{\star}>0$ such that $I(u)>0$ for $0<t<t^{\star}$ and $I\left(u\left(t^{\star}\right)\right)=0$, then there exists a weak solution $u(x, t)$ which vanishes in finite time $t^{\star}$.

Theorem 1.4. Let conditions $(a)-(d)$ hold, $u_{0} \in X_{0}$. If $J\left(u_{0}\right)=d$ and $I\left(u_{0}\right)<0$, then there exists a finite time $T^{\star}$ such that the weak solution of problem (1.1) blows up in the sense of (1.6).

To state the main results regarding the high initial energy $\left(J\left(u_{0}\right)>d\right)$, we introduce the following sets:

$$
\begin{aligned}
\mathcal{B} & =\left\{u_{0} \in X_{0} \mid \text { the solution } u \text { of problem (1.1) blows up in finite time }\right\} \\
\mathcal{G}_{0} & =\left\{u_{0} \in X_{0} \mid u(t) \rightarrow 0 \text { in } X_{0} \text { as } t \rightarrow \infty\right\}
\end{aligned}
$$

Theorem 1.5. Let conditions $(a)-(d)$ hold and $u_{0} \in X_{0}$. If $J\left(u_{0}\right)>d$, then the following statements hold:

(1) If $u_{0} \in \mathcal{N}_{+}$and $\left\|u_{0}\right\|_{L^{2}(\Omega)} \leqslant \lambda_{J\left(u_{0}\right)}$, then $u_{0} \in \mathcal{G}_{0}$.

(2) If $u_{0} \in \mathcal{N}_{-}$and $\left\|u_{0}\right\|_{L^{2}(\Omega)} \geqslant \Lambda_{J\left(u_{0}\right)} \lambda_{J\left(u_{0}\right)}$, then $u_{0} \in \mathcal{B}$.

The following theorem indicates that there exists blow-up solutions to problem (1.1) for any high initial energy.

Theorem 1.6. Let conditions $(a)-(d)$ hold and $u_{0} \in X_{0}$. For any $M>d$, then there exists $u_{M} \in \mathcal{N}_{-}$such that $J\left(u_{M}\right)=M$ and $u_{M} \in \mathcal{B}$.

This paper is organized as follows. Some necessary definitions and properties of the functional spaces used in this paper are given in Section 2. In Section 3, we define some notations and give some results about potential well theory. Section 4 and Section 5 are devoted to the cases $J\left(u_{0}\right)<d$ and $J\left(u_{0}\right)=d$, respectively. In Section 6 , we consider the case $J\left(u_{0}\right)>d$.

\section{Functional Space and Preliminaries}

In this section, let's recall some necessary definitions and properties of the functional spaces introduced by R. Servadei and E. Valdinoci in [26, 27]. Let $0<s<1,1<p<\infty$ be real numbers and the fractional critical exponent $p_{s}^{\star}$ be defined as $p_{s}^{\star}=\frac{N p}{N-s p}$ if $s p<N$ and $p_{s}^{\star}=\infty$, otherwise. Define the Banach space

$$
W^{s, p}\left(\mathbb{R}^{N}\right)=\left\{u \in L^{p}\left(\mathbb{R}^{N}\right), \frac{|u(x)-u(y)|}{|x-y|^{\frac{N}{p}+s}} \in L^{p}\left(\mathbb{R}^{N} \times \mathbb{R}^{N}\right)\right\}
$$

endowed with the norm

$$
\|u\|_{W^{s, p}\left(\mathbb{R}^{N)}\right.}=\|u\|_{L^{p}\left(\mathbb{R}^{N)}\right.}+\left(\int_{\mathbb{R}^{N}} \int_{\mathbb{R}^{N}} \frac{|u(x)-u(y)|^{p}}{|x-y|^{N+s p}} \mathrm{~d} y \mathrm{~d} x\right)^{\frac{1}{p}} .
$$

Let $\Omega$ an open set in $\mathbb{R}^{N}$ and $Q=\left(\mathbb{R}^{N} \times \mathbb{R}^{N}\right) \backslash(\mathcal{Q} \Omega \times \varrho \Omega), \mathcal{C} \Omega=\mathbb{R}^{N} \backslash \Omega$. It is obvious that $\Omega \times \Omega$ is strictly contained in $Q$. Denote

$$
X_{0}(\Omega)=\left\{u \mid u \in L^{p}(\Omega), u=0 \text { in } \Theta \Omega, \frac{|u(x)-u(y)|}{|x-y|^{\frac{N}{p}+s}} \in L^{p}(Q)\right\} .
$$

The space $X_{0}$ is a normed linear subspace of $W^{s, p}\left(\mathbb{R}^{N}\right)$ and also endowed with the norm

$$
\|u\|_{W^{s, p}\left(\mathbb{R}^{N)}\right.}=\|u\|_{L^{p}(\Omega)}+\left(\iint_{Q} \frac{|u(x)-u(y)|^{p}}{|x-y|^{N+p s}} \mathrm{~d} y \mathrm{~d} x\right)^{\frac{1}{p}} .
$$


It is known that $\left(X_{0},\|\cdot\|_{X_{0}}\right)$ is a uniformly convex reflexive Banach space, and its norm is equivalent to

$$
\|u\|_{X_{0}}=\left(\iint_{Q} \frac{|u(x)-u(y)|^{p}}{|x-y|^{N+p s}} \mathrm{~d} y \mathrm{~d} x\right)^{\frac{1}{p}} .
$$

We list two useful lemmas used in the sequel. For more properties of the space $X_{0}(\Omega)$, readers could refer to $[5,26,27]$.

Lemma 2.1. [5, Theorem 2.4] For any $s>0, C_{0}^{\infty}(\Omega)$ of smooth functions with compact support is a dense subspace of $X_{0}$.

Lemma 2.2. [5, Theorem 6.5] Let $\left\{u_{k}\right\}$ be a bounded sequence in $X_{0}$. Then there exists $u \in L^{\beta}\left(\mathbb{R}^{N}\right)$ such that up to a subsequence, $u_{k} \rightarrow u$ strongly in $L^{\beta}(\Omega)$ as $k \rightarrow \infty$ for any $\beta \in\left[1, p_{s}^{\star}\right)$.

At the end of this section, we list some properties about the nonlinear term in the problem (1.1). These lemma and corollary were obtained in [23].

Lemma 2.3. If $f$ satisfy conditions $(a)-(c)$. Then

(1) $|F(u)| \leqslant A|u|^{r}$ for some $A>0$ and all $u \in \mathbb{R}$.

(2) $F(u) \geqslant B|u|^{q+1}$ for some $B>0$ and $|u| \geqslant 1$.

(3) $u\left(u f^{\prime}(u)-f(u)\right) \geqslant 0$, the equality holds only for $u=0$.

Corollary 2.1. Under the conditions of Lemma 2.3, we have

(1) $|u f(u)| \leqslant r A|u|^{r},|f(u)| \leqslant r A|u|^{r-1}$ for all $u \in \mathbb{R}$.

(2) $u f(u) \geqslant(q+1) B|u|^{q+1}$ for $|u| \geqslant 1$.

\section{Preliminaries of potential well}

Throughout this paper, $C_{\star}$ is the optimal embedding constant from $X_{0}$ into $L^{r}(\Omega)$ with $r \in\left(1, p_{s}^{\star}\right)$. To state our results, we need to introduce some notations and definitions of some functionals and sets.

For $u \in X_{0}(\Omega)$, set

$$
\begin{aligned}
& J(u)=\frac{1}{p}\|u\|_{X_{0}}^{p}-\int_{\Omega} F(u) \mathrm{d} x ; \\
& I(u)=\|u\|_{X_{0}}^{p}-\int_{\Omega} u f(u) \mathrm{d} x,
\end{aligned}
$$

and define the Nehari manifold

$$
\mathcal{N}=\left\{u \in X_{0} \mid I(u)=0,\|u\|_{X_{0}} \neq 0\right\} .
$$

If conditions $(a)-(c)$ hold, then functionals $J$ and $I$ are well-defined and continuous on $X_{0}(\Omega)$, which can be proved by the similar argument for Lemma 3.1 and Lemma 3.2 in [33]. The potential well and its corresponding set are defined respectively by

$$
\begin{aligned}
& W=\left\{u \in X_{0} \mid I(u)>0, J(u)<d\right\} \cup\{0\} ; \\
& V=\left\{u \in X_{0} \mid I(u)<0, J(u)<d\right\},
\end{aligned}
$$

where

$$
d=\inf _{0 \neq u \in X_{0}} \sup _{\lambda \geqslant 0} J(\lambda u)=\inf _{u \in \mathcal{N}} J(u)
$$

is the depth of the potential well $W$.

The following lemmas follow the line of [18], but there exist some differences because of the generality of $f(u)$ and existence of $(-\Delta)_{p}^{s} u$. Hence, we will give the specific process of proof. 
Lemma 3.1. The depth of the potential well $W$ is positive.

Proof. Fix $u \in \mathcal{N}$, from Corollary 2.1(1) and the embedding $X_{0}$ into $L^{r}(\Omega)$, we get

$$
\|u\|_{X_{0}}^{p}=\int_{\Omega} u f(u) \mathrm{d} x \leqslant A r\|u\|_{L^{r}(\Omega)}^{r} \leqslant A r C_{\star}^{r}\|u\|_{X_{0}}^{r},
$$

which implies $\|u\|_{X_{0}} \geqslant\left(\frac{1}{A r C_{\star}^{r}}\right)^{\frac{1}{r-p}}$. By the condition (c), we have

$$
\begin{aligned}
J(u) & \geqslant \frac{1}{p}\|u\|_{X_{0}}^{p}-\frac{1}{q+1} \int_{\Omega} u f(u) \mathrm{d} x \\
& =\frac{1}{p}\|u\|_{X_{0}}^{p}-\frac{1}{q+1}\|u\|_{X_{0}}^{p} \\
& =\frac{q+1-p}{p(q+1)}\|u\|_{X_{0}}^{p} \\
& \geqslant \frac{q+1-p}{p(q+1)}\left(\frac{1}{A r C_{\star}^{r}}\right)^{\frac{p}{r-p}} .
\end{aligned}
$$

Therefore $d \geqslant \frac{q+1-p}{p(q+1)}\left(\frac{1}{\operatorname{ArC} C_{\star}^{r}}\right)^{\frac{p}{r-p}}>0$.

Lemma 3.2. If $I(u)>0$, then $J(u)>0$.

Proof. Assuming that $J(u) \leqslant 0$, by a direct computation, we get

$$
\int_{\Omega} u f(u) \mathrm{d} x<\|u\|_{X_{0}}^{p} \leqslant p \int_{\Omega} F(u) \mathrm{d} x .
$$

According to condition (c), we get

$$
p \int_{\Omega} F(u) \mathrm{d} x \leqslant \frac{p}{q+1} \int_{\Omega} u f(u) \mathrm{d} x .
$$

It is obvious from $p<q+1$ that there does not exist $\|u\|_{X_{0}}^{p}$ satisfying (3.1). Therefore, this contradiction implies the assumption $J(u) \leqslant 0$ does not hold.

For any $\delta>0$, define the modified functional and Nehari manifold as follows:

$$
\begin{aligned}
& I_{\delta}(u)=\delta\|u\|_{X_{0}}^{p}-\int_{\Omega} u f(u) \mathrm{d} x ; \\
& \mathcal{N}_{\delta}=\left\{u \in X_{0} \mid I_{\delta}(u)=0,\|u\|_{X_{0}} \neq 0\right\} .
\end{aligned}
$$

The corresponding modified potential well and its corresponding set are defined respectively by

$$
\begin{aligned}
& W_{\delta}=\left\{u \in X_{0} \mid I_{\delta}(u)>0, J(u)<d(\delta)\right\} \cup\{0\} ; \\
& V_{\delta}=\left\{u \in X_{0} \mid I_{\delta}(u)<0, J(u)<d(\delta)\right\},
\end{aligned}
$$

where $d(\delta)=\inf _{u \in \mathcal{N}_{\delta}} J(u)$ is the potential depth of $W_{\delta}$.

Define

$$
\begin{aligned}
& \mathcal{N}_{+}=\left\{u \in X_{0} \mid I(u)>0\right\} ; \\
& \mathcal{N}_{-}=\left\{u \in X_{0} \mid I(u)<0\right\},
\end{aligned}
$$

and the (open) sublevels of $J$

$$
J^{\varsigma}=\left\{u \in X_{0} \mid J(u)<\varsigma\right\}
$$


Obviously, by the definition of $J(u), \mathcal{N}, J^{\varsigma}$ and $d$, we get

$$
\mathcal{N}^{\varsigma}:=\mathcal{N} \cap J^{\varsigma}=\left\{u \in \mathcal{N} \mid \frac{1}{p}\|u\|_{X_{0}}^{p}-\int_{\Omega} F(u) \mathrm{d} x<\varsigma\right\} \neq \emptyset, \quad \forall \varsigma>d .
$$

For $\varsigma>d$, define

$$
\lambda_{\varsigma}=\inf \left\{\|u\|_{L^{2}(\Omega)} \mid u \in \mathcal{N}^{\varsigma}\right\}, \Lambda_{\varsigma}=\sup \left\{\|u\|_{L^{2}(\Omega)} \mid u \in \mathcal{N}^{\varsigma}\right\} .
$$

Obviously, $\lambda_{\varsigma}$ is non-increasing and $\Lambda_{\zeta}$ is non-decreasing.

Lemma 3.3. For any $u \in X_{0}$ with $\|u\|_{X_{0}} \neq 0$, we have

(1) $\lim _{\lambda \rightarrow 0^{+}} J(\lambda u)=0, \lim _{\lambda \rightarrow+\infty} J(\lambda u)=-\infty$.

(2) There exists a unique $\lambda^{\star}=\lambda^{\star}(u)>0$ such that $\left.\frac{\mathrm{d} J(\lambda u)}{\mathrm{d} \lambda}\right|_{\lambda=\lambda^{\star}}=0$. $J(\lambda u)$ is increasing on $0<\lambda \leqslant \lambda^{\star}$, decreaing on $\lambda^{\star} \leqslant \lambda<\infty$ and take its maximum at $\lambda=\lambda^{\star}$.

(3) $I(\lambda u)>0$ on $0<\lambda \leqslant \lambda^{\star}, I(\lambda u)<0$ on $\lambda^{\star} \leqslant \lambda<\infty$ and $I\left(\lambda^{*} u\right)=0$.

Proof. (1) It follows from the definition of $J(u)$, condition $(c)$ and Corollary 2.1(1) that

$$
\begin{aligned}
J(\lambda u) & =\frac{\lambda^{p}}{p}\|u\|_{X_{0}}^{p}-\int_{\Omega} F(\lambda u) \mathrm{d} x \\
& \geqslant \frac{\lambda^{p}}{p}\|u\|_{X_{0}}^{p}-\frac{1}{q+1} \int_{\Omega} \lambda u f(\lambda u) \mathrm{d} x \\
& \geqslant \frac{\lambda^{p}}{p}\|u\|_{X_{0}}^{p}-\frac{A r \lambda^{r}}{q+1}\|u\|_{L^{r}(\Omega)}^{r} .
\end{aligned}
$$

Obviously, assertion (1) follows from $r>p$. we get

(2) Clearly, $J(\lambda u)=0$ at $\lambda=0$, and $J(\lambda u)>0$ for any $\lambda \in\left(0,\left(\frac{q+1}{A r p}\|u\|_{X_{0}}^{p} \|_{L^{r}(\Omega)}^{\frac{1}{r-p}}\right)\right.$. By a direct computation,

$$
\frac{\mathrm{d} J(\lambda u)}{\mathrm{d} \lambda}=\lambda^{p-1}\|u\|_{X_{0}}^{p}-\int_{\Omega} f(\lambda u) u \mathrm{~d} x .
$$

Therefore, there exists a $\lambda^{\star}>0$ such that

$$
\left.\frac{\mathrm{d} J(\lambda u)}{\mathrm{d} \lambda}\right|_{\lambda=\lambda^{\star}}=\left(\lambda^{\star}\right)^{p-1}\|u\|_{X_{0}}^{p}-\int_{\Omega} f\left(\lambda^{\star} u\right) u \mathrm{~d} x=0 .
$$

Moreover, combining with condition $(d)$ and $\|u\|_{X_{0}} \neq 0$, we get

$$
\begin{aligned}
\left.\frac{\mathrm{d}^{2} J(\lambda u)}{\mathrm{d} \lambda^{2}}\right|_{\lambda=\lambda^{\star}} & =(p-1)\left(\lambda^{\star}\right)^{p-2}\|u\|_{X_{0}}^{p}-\int_{\Omega} f^{\prime}\left(\lambda^{\star} u\right) u^{2} \mathrm{~d} x \\
& =\frac{1}{\left(\lambda^{\star}\right)^{2}}\left((p-1)\left(\lambda^{\star}\right)^{p}\|u\|_{X_{0}}^{p}-\int_{\Omega} f^{\prime}\left(\lambda^{\star} u\right)\left(\lambda^{\star} u\right)^{2} \mathrm{~d} x\right) \\
& =\frac{1}{\left(\lambda^{\star}\right)^{2}}\left((p-1) \int_{\Omega} f\left(\lambda^{\star} u\right) \lambda^{\star} u \mathrm{~d} x-\int_{\Omega} f^{\prime}\left(\lambda^{\star} u\right)\left(\lambda^{\star} u\right)^{2} \mathrm{~d} x\right) \\
& <0 .
\end{aligned}
$$

Next, we prove that $\lambda^{\star}=\lambda^{\star}(u)$ is uniquely determined. Assume that there exists two roots $\lambda_{1}, \lambda_{2}$ of $\frac{d J(\lambda u)}{d \lambda}=0$. That is

$$
\left.\frac{\mathrm{d} J(\lambda u)}{\mathrm{d} \lambda}\right|_{\lambda=\lambda_{1}}=0,\left.\frac{\mathrm{d}^{2} J(\lambda u)}{\mathrm{d} \lambda^{2}}\right|_{\lambda=\lambda_{1}}<0
$$




$$
\left.\frac{\mathrm{d} J(\lambda u)}{\mathrm{d} \lambda}\right|_{\lambda=\lambda_{2}}=0,\left.\frac{\mathrm{d}^{2} J(\lambda u)}{\mathrm{d} \lambda^{2}}\right|_{\lambda=\lambda_{2}}<0 .
$$

If there exists a $\lambda_{3}$ with $\lambda_{1}<\lambda_{3}<\lambda_{2}$ such that $J\left(\lambda_{3} u\right)$ is the minimum of $J(\lambda u)$ in $\left[\lambda_{1}, \lambda_{2}\right]$, then

$$
\left.\frac{\mathrm{d} J(\lambda u)}{\mathrm{d} \lambda}\right|_{\lambda=\lambda_{3}}=0,\left.\frac{\mathrm{d}^{2} J(\lambda u)}{\mathrm{d} \lambda^{2}}\right|_{\lambda=\lambda_{3}} \geqslant 0,
$$

which is a contradiction. Therefore, we conclude that $J(\lambda u)$ gets its minimum in $\left[\lambda_{1}, \lambda_{2}\right]$ at either $\lambda_{1}$ or $\lambda_{2}$. If the first case happens, then there exists a $\sigma>0$ such that $J(\lambda u)$ at $\left[\lambda_{1}, \lambda_{1}+\sigma\right]$ is not only maximum, but also minimum. That is, $J(\lambda u)$ is a constant. Therefore, we obtain

$$
\left.\frac{\mathrm{d} J(\lambda u)}{\mathrm{d} \lambda}\right|_{\lambda=\lambda_{1}}=0,\left.\frac{\mathrm{d}^{2} J(\lambda u)}{\mathrm{d} \lambda^{2}}\right|_{\lambda=\lambda_{1}}=0
$$

which contradicts (3.2). We conclude that $J\left(\lambda_{1} u\right)$ is not the minimum. By the same argument, $J\left(\lambda_{2} u\right)$ also is not the minimum. So we complete the proof of (2).

Next, we will prove (3). By the definition of $I(u)$, we get

$$
\frac{\mathrm{d} J(\lambda u)}{\mathrm{d} \lambda}=\lambda^{p-1}\|u\|_{X_{0}}^{p}-\int_{\Omega} f(\lambda u) u \mathrm{~d} x=\frac{I(\lambda u)}{\lambda} .
$$

It is obvious that (3) holds from (2).

Lemma 3.4. For any $u \in X_{0}$ and $y(\delta)=\left(\frac{\delta}{A r C_{*}^{r}}\right)^{\frac{1}{r-p}}$, we have

(1) If $0 \leqslant\|u\|_{X_{0}} \leqslant y(\delta)$, then $I_{\delta}(u) \geqslant 0$.

(2) If $I_{\delta}(u)<0$, then $\|u\|_{X_{0}}>y(\delta)$.

(3) If $I_{\delta}(u)=0$, then $\|u\|_{X_{0}}=0$ or $\|u\|_{X_{0}} \geqslant y(\delta)$.

Proof. (1) We get

$$
\int_{\Omega} u f(u) \mathrm{d} x \leqslant r A\|u\|_{L^{r}(\Omega)}^{r} \leqslant A r C_{\star}^{r}\|u\|_{X_{0}}^{r} \leqslant \operatorname{ArC} C_{\star}^{r}(y(\delta))^{r-p}\|u\|_{X_{0}}^{p}
$$

from Corollary 2.1(1) and the embedding from $X_{0}$ into $L^{r}(\Omega)$. So it implies $I_{\delta}(u) \geqslant 0$.

(2) It easily follows from (1).

(3) If $\|u\|_{X_{0}}=0$, then $I_{\delta}(u)=0$. If $I_{\delta}(u)=0,\|u\|_{X_{0}} \neq 0$, then from Corollary 2.1(1) and the embedding from $X_{0}$ into $L^{r}(\Omega)$, we have

$$
\delta\|u\|_{X_{0}}^{p}=\int_{\Omega} u f(u) \mathrm{d} x \leqslant r A\|u\|_{L^{r}(\Omega)}^{r} \leqslant A r C_{\star}^{r}\|u\|_{X_{0}}^{r-p}\|u\|_{X_{0}}^{p},
$$

which implies $\|u\|_{X_{0}} \geqslant y(\delta)$.

Lemma 3.5. The function $d(\delta)$ satisfies the following properties:

(1) $\lim _{\delta \rightarrow 0^{+}} d(\delta)=0, \lim _{\delta \rightarrow+\infty} d(\delta)=-\infty$.

(2) $d(\delta)$ is strictly increasing on $0<\delta \leqslant 1$, strictly decreasing on $\delta \geqslant 1$ and take its maximum $d=d(1)$ at $\delta=1$.

Proof. (1) For any $u \in X_{0}$ with $\|u\|_{X_{0}} \neq 0$ and $\delta>0$, there exists a unique $\lambda=\lambda(\delta)$ such that $I_{\delta}(\lambda u)=0$ from Lemma 3.3(3). Furthermore, we get

$$
\delta \lambda^{p}\|u\|_{X_{0}}^{p}=\int_{\Omega} \lambda u f(\lambda u) \mathrm{d} x
$$

It is obvious that

$$
\int_{\Omega} \lambda u f(\lambda u) \mathrm{d} x=\lambda^{p} \eta(\lambda),
$$


where

$$
\eta(\lambda):=\frac{1}{\lambda^{p-1}} \int_{\Omega} u f(\lambda u) \mathrm{d} x
$$

We have the following two claims:

Claim 1: $\eta(\lambda)$ is strictly increasing on $(0,+\infty)$.

Indeed, under the condition $(\mathrm{d})$, we get

$$
\eta^{\prime}(\lambda)=\frac{1}{\lambda^{p+1}}\left(\int_{\Omega}(\lambda u)^{2} f^{\prime}(\lambda u) \mathrm{d} x-\int_{\Omega}(p-1) \lambda u f(\lambda u) \mathrm{d} x\right)>0,
$$

which yields Claim 1.

Claim 2: $\lim _{\lambda \rightarrow 0^{+}} \eta(\lambda)=0, \lim _{\lambda \rightarrow+\infty} \eta(\lambda)=+\infty$.

By Corollary 2.1(1), we get

$$
\eta(\lambda)=\frac{1}{\lambda^{p-1}} \int_{\Omega} u f(\lambda u) \mathrm{d} x \leqslant r A \lambda^{r-p}\|u\|_{L^{r}(\Omega)}^{r}
$$

Therefore, $\lim _{\lambda \rightarrow 0^{+}} \eta(\lambda)=0$. For sufficiently large $\lambda$, we obtain

$$
\eta(\lambda)=\frac{1}{\lambda^{p-2}} \int_{\Omega} \lambda u f(\lambda u) \mathrm{d} x \geqslant \frac{(q+1) B}{\lambda^{p-2}}\|\lambda u\|_{L^{q+1}(\Omega)}^{q+1}=(q+1) B \lambda^{q-p+3}\|u\|_{L^{q+1}(\Omega)}^{q+1}
$$

by Corollary 2.1(2). It is obvious that $\lim _{\lambda \rightarrow+\infty} \eta(\lambda)=+\infty$. This completes the proof of Claim 2 .

Therefore, it follows from Claim 1, Claim 2 and (3.3) that $\lambda=\eta^{-1}\left(\delta\|u\|_{X_{0}}^{p}\right)$, that is, inverse function of $\eta$, then $\lambda$ is strictly increasing on $(0,+\infty)$ and

$$
\lim _{\delta \rightarrow 0^{+}} \lambda(\delta)=0, \quad \lim _{\delta \rightarrow+\infty} \lambda(\delta)=+\infty,
$$

Since $\lambda u \in \mathcal{N}_{\delta}, d(\delta) \leqslant J(\lambda u)$, combining with Lemma 3.3(1), we get

$$
0 \leqslant \lim _{\delta \rightarrow 0^{+}} d(\delta) \leqslant \lim _{\delta \rightarrow 0^{+}} J(\lambda u)=\lim _{\lambda \rightarrow 0^{+}} J(\lambda u)=0,
$$

and

$$
\lim _{\delta \rightarrow+\infty} d(\delta) \leqslant \lim _{\delta \rightarrow+\infty} J(\lambda u)=\lim _{\lambda \rightarrow+\infty} J(\lambda u)=-\infty .
$$

This completes the proof of (1).

(2) Clearly, we only need to prove that any $0<\delta^{\prime}<\delta^{\prime \prime}<1$ or $\delta^{\prime}>\delta^{\prime \prime}>1$ and any $u \in \mathcal{N}_{\delta^{\prime \prime}}$, there exists a $v \in \mathcal{N}_{\delta^{\prime}}$ and a constant $\epsilon\left(\delta^{\prime}, \delta^{\prime \prime}\right)$ such that $J(u)-J(v) \geqslant \epsilon\left(\delta^{\prime}, \delta^{\prime \prime}\right)$. Actually, for $u \in \mathcal{N}_{\delta^{\prime \prime}}$, we get $I_{\delta^{\prime \prime}}(u)=0$ (This implies $\lambda\left(\delta^{\prime \prime}\right)=1$ from (3.3)) and $\|u\|_{X_{0}} \geqslant y\left(\delta^{\prime \prime}\right)$ by Lemma 3.4(3). By the definition of $\lambda$ in (3.3), then $I_{\delta}(\lambda u)=0$. Therefore, choosing $v=\lambda\left(\delta^{\prime}\right) u$, then we have $v \in \mathcal{N}_{\delta^{\prime}}$. Let $g(\lambda)=J(\lambda(\delta) u)$, then

$$
\begin{aligned}
\frac{\mathrm{d} g(\lambda)}{\mathrm{d} \lambda} & =\lambda^{p-1}\|u\|_{X_{0}}^{p}-\int_{\Omega} f(\lambda u) u \mathrm{~d} x \\
& =\frac{1}{\lambda}\left((1-\delta)\|\lambda u\|_{X_{0}}^{p}+I_{\delta}(\lambda u)\right) \\
& =\lambda^{p-1}(1-\delta)\|u\|_{X_{0}}^{p} .
\end{aligned}
$$


If $0<\delta^{\prime}<\delta^{\prime \prime}<1$, since $\lambda$ is strictly increasing (Claim 2) and $\lambda\left(\delta^{\prime \prime}\right)=1$, then

$$
\begin{aligned}
J(u)-J(v) & =g(1)-g\left(\lambda\left(\delta^{\prime}\right)\right)=\int_{\lambda\left(\delta^{\prime}\right)}^{1} \frac{\mathrm{d} g(\lambda u)}{\mathrm{d} \lambda} \mathrm{d} \lambda \\
& =\int_{\lambda\left(\delta^{\prime}\right)}^{1} \lambda^{p-1}(1-\delta)\|u\|_{X_{0}}^{p} \mathrm{~d} \lambda \\
& \geqslant \frac{1}{p}\left(1-\delta^{\prime \prime}\right)\left(y\left(\delta^{\prime \prime}\right)\right)^{p}\left(\lambda\left(\delta^{\prime}\right)\right)^{p}\left(1-\lambda\left(\delta^{\prime}\right)\right) \\
& =\epsilon\left(\delta^{\prime}, \delta^{\prime \prime}\right)>0 .
\end{aligned}
$$

if $\delta^{\prime}>\delta^{\prime \prime}>1$, then

$$
\begin{aligned}
J(u)-J(v) & =\int_{\lambda\left(\delta^{\prime}\right)}^{1} \frac{\mathrm{d} g(\lambda u)}{\mathrm{d} \lambda} \mathrm{d} \lambda \\
& =-\int_{1}^{\lambda\left(\delta^{\prime}\right)} \lambda^{p-1}(1-\delta)\|u\|_{X_{0}}^{p} \mathrm{~d} \lambda \\
& \geqslant \frac{1}{p}\left(\delta^{\prime \prime}-1\right)\left(y\left(\delta^{\prime \prime}\right)\right)^{p}\left(\lambda\left(\delta^{\prime \prime}\right)\right)^{p}\left(\lambda\left(\delta^{\prime}\right)-1\right) \\
& =\epsilon\left(\delta^{\prime}, \delta^{\prime \prime}\right)>0 .
\end{aligned}
$$

Since $d(\delta)$ is continuous, it is obvious that it take its maximum $d=d(1)$ at $\delta=1$.

Lemma 3.6. Assume $u \in X_{0}, 0<J(u)<d$ and $\delta_{1}<1<\delta_{2}, \delta_{1}, \delta_{2}$ satisfy the equation $d(\delta)=J(u)$. Then the sign of $I_{\delta}(u)$ does not change for $\delta_{1}<\delta<\delta_{2}$.

Proof. Clearly, $J(u)>0$ implies $\|u\|_{X_{0}} \neq 0$. If the sign of $I_{\delta}(u)$ changes for $\delta_{1}<\delta<\delta_{2}$, then there exists a $\bar{\delta} \in\left(\delta_{1}, \delta_{2}\right)$ such that $I_{\bar{\delta}}(u)=0$. Thus by the definition of $d(\delta)$ we get $J(u) \geqslant d(\bar{\delta})$, which contradicts $J(u)=d\left(\delta_{1}\right)=d\left(\delta_{2}\right)<d(\bar{\delta})$ by Lemma 3.5(2).

For $\varphi \in W^{s, p}\left(\mathbb{R}^{N}\right)$, due to the symmetry of the kernel, we have the following equality:

$$
\begin{aligned}
\int_{\mathbb{R}^{N}}(-\Delta)_{p}^{s} u(x) \varphi(x) \mathrm{d} x & =\int_{\mathbb{R}^{N}} \int_{\mathbb{R}^{N}} \frac{|u(x)-u(y)|^{p-2}(u(x)-u(y))}{|x-y|^{N+p s}} \varphi(x) \mathrm{d} y \mathrm{~d} x \\
& =-\frac{1}{2} \int_{\mathbb{R}^{N}} \int_{\mathbb{R}^{N}} \frac{|u(x)-u(y)|^{p-2}(u(x)-u(y))}{|x-y|^{N+p s}}(\varphi(x)-\varphi(y)) \mathrm{d} y \mathrm{~d} x,
\end{aligned}
$$

which leads to the following definition of weak solutions by using $u(x)=0$ in $\mathrm{C} \Omega$.

Definition 3.1. A function $u=u(x, t) \in L^{\infty}\left(0, T ; X_{0}\right)$ with $u_{t} \in L^{2}\left(0, T ; L^{2}(\Omega)\right)$ is called a weak solution to problem (1.1) if $u(x, 0)=u_{0}(x)$ and the following equality holds

$$
\left(u_{t}, \varphi\right)_{L^{2}(\Omega)}+(u, \varphi)_{X_{0}}=(f(u), \varphi)_{L^{2}(\Omega)}, \quad \text { a.e. } t>0,
$$

here

$$
(u, \varphi)_{X_{0}}=\frac{1}{2} \iint_{Q} \frac{|u(y)-u(x)|^{p-2}(u(y)-u(x))}{|x-y|^{N+p s}}(\varphi(y)-\varphi(x)) \mathrm{d} y \mathrm{~d} x
$$

for all $\varphi \in X_{0}$. Moreover,

$$
\int_{0}^{t}\left\|u_{\tau}\right\|_{L^{2}(\Omega)}^{2} \mathrm{~d} \tau+J(u)=J\left(u_{0}\right), \quad \text { a.e. } t>0 .
$$


Lemma 3.7. Assume that $u$ is a weak solution of problem (1.1) with $0<J\left(u_{0}\right)<d$ and $\delta_{1}<1<\delta_{2}, \delta_{1}, \delta_{2}$ satisfy the equation $d(\delta)=J\left(u_{0}\right)$.

(1) If I $\left(u_{0}\right)>0$, then $u(x, t) \in W_{\delta}$ for $\delta_{1}<\delta<\delta_{2}$ and $0<t<T$.

(2) If I $\left(u_{0}\right)<0$, then $u(x, t) \in V_{\delta}$ for $\delta_{1}<\delta<\delta_{2}$ and $0<t<T$.

Proof. (1) For $0<J\left(u_{0}\right)=d\left(\delta_{1}\right)=d\left(\delta_{2}\right)<d$ and $I\left(u_{0}\right)>0$, from Lemma 3.6, we get $u_{0} \in W_{\delta}$ for $\delta_{1}<\delta<\delta_{2}$. Next we prove $u(x, t) \in W_{\delta}$ for $\delta_{1}<\delta<\delta_{2}$ and $0<t<T$. Otherwise, there exists a $t_{0} \in(0, T)$ and a $\delta_{0} \in\left(\delta_{1}, \delta_{2}\right)$ such that $u\left(t_{0}\right) \in \partial W_{\delta_{0}}$, then

$$
I_{\delta_{0}}\left(u\left(t_{0}\right)\right)=0,\left\|u\left(t_{0}\right)\right\|_{X_{0}} \neq 0 \text { or } J\left(u\left(t_{0}\right)\right)=d\left(\delta_{0}\right) .
$$

Clearly, $J\left(u\left(t_{0}\right)\right)<d\left(\delta_{0}\right)$ from (3.5). Thus $I_{\delta_{0}}\left(u\left(t_{0}\right)\right)=0,\left\|u\left(t_{0}\right)\right\|_{X_{0}} \neq 0$, the definition of $d\left(\delta_{0}\right)$ implies $J\left(u\left(t_{0}\right)\right) \geqslant d\left(\delta_{0}\right)$, which contradicts (3.5).

(2) Similarly, $u_{0} \in V_{\delta}$ for $\delta_{1}<\delta<\delta_{2}$. Next we show $u(x, t) \in V_{\delta}$ for $\delta_{1}<\delta<\delta_{2}$ and $0<t<T$. If not, there exists a $t_{0} \in(0, T)$ and a $\delta_{0} \in\left(\delta_{1}, \delta_{2}\right)$ such that $u\left(t_{0}\right) \in \partial V_{\delta_{0}}$, then

$$
I_{\delta_{0}}\left(u\left(t_{0}\right)\right)=0 \text { or } J\left(u\left(t_{0}\right)\right)=d\left(\delta_{0}\right) .
$$

Clearly, $I_{\delta_{0}}\left(u\left(t_{0}\right)\right)=0$. Assuming $t_{0}$ is the first time such that $I_{\delta_{0}}\left(u\left(t_{0}\right)\right)=0$, the $I_{\delta_{0}}(u(t))<0$ for $0 \leqslant t<t_{0}$. By Lemma 3.4(2), we get $\|u\|_{X_{0}}>y(\delta)$ for $0 \leqslant t<t_{0}$. Lemma 3.4(3) implies $\left\|u\left(t_{0}\right)\right\|_{X_{0}} \geqslant y\left(\delta_{0}\right)$, combining with $I_{\delta_{0}}\left(u\left(t_{0}\right)\right)=0$, we get $u\left(t_{0}\right) \in \mathcal{N}_{\delta_{0}}$. By the definition of $d\left(\delta_{0}\right)$, we get $J\left(u\left(t_{0}\right)\right) \geqslant d\left(\delta_{0}\right)$, which contradicts (3.5).

Lemma 3.8. If conditions $(a)-(c)$ hold, then

(1) 0 is away from both $\mathcal{N}$ and $\mathcal{N}_{-}$, that is, $\operatorname{dist}(0, \mathcal{N})>0$ and $\operatorname{dist}\left(0, \mathcal{N}_{-}\right)>0$.

(2) For any $\varsigma>0$, the set $J^{\varsigma} \cap \mathcal{N}_{+}$is bounded in $X_{0}$.

Proof. (1) For $u \in \mathcal{N}$, by the definition of $d$, Lemma 2.3(1) and the embedding from $X_{0}$ into $L^{r}(\Omega)$, we get

$$
d \leqslant \frac{1}{p}\|u\|_{X_{0}}^{p}-\int_{\Omega} F(u) \mathrm{d} x \leqslant \frac{1}{p}\|u\|_{X_{0}}^{p}+A\|u\|_{L^{r}(\Omega)}^{r} \leqslant \frac{1}{p}\|u\|_{X_{0}}^{p}+A C_{*}^{r}\|u\|_{X_{0}}^{r},
$$

which implies that there exists a constant $\rho>0$ such that $\operatorname{dist}(0, \mathcal{N})=\inf _{u \in \mathcal{N}}\|u\|_{X_{0}} \geqslant \rho$.

For $u \in \mathcal{N}_{-}$, we get $\|u\|_{X_{0}} \neq 0$. Then the embedding from $X_{0}$ into $L^{r}(\Omega)$ implies that

$$
\|u\|_{X_{0}}^{p}<\int_{\Omega} u f(u) \mathrm{d} x \leqslant r A\|u\|_{L^{r}(\Omega)}^{r} \leqslant A r C_{\star}^{r}\|u\|_{X_{0}}^{r},
$$

which implies $\|u\|_{X_{0}}>\left(\frac{1}{A r C_{*}^{r}}\right)^{\frac{1}{r-p}}$. Furthermore, $\operatorname{dist}\left(0, \mathcal{N}_{-}\right)=\inf _{u \in \mathcal{N}_{-}}\|u\|_{X_{0}}>0$.

(2) For any $u \in J^{\varsigma} \cap \mathcal{N}_{+}$, then $J(u)<\varsigma$ and $I(u)>0$. Therefore, from condition (c), we get

$$
\begin{aligned}
\varsigma>J(u) & \geqslant \frac{1}{p}\|u\|_{X_{0}}^{p}-\frac{1}{q+1} \int_{\Omega} u f(u) \mathrm{d} x \\
& =\frac{q+1-p}{p(q+1)}\|u\|_{X_{0}}^{p}+\frac{1}{q+1} I(u) \\
& >\frac{q+1-p}{p(q+1)}\|u\|_{X_{0}}^{p},
\end{aligned}
$$

which yields $\|u\|_{X_{0}}<\left(\frac{p(q+1) \zeta}{q+1-p}\right)^{\frac{1}{p}}$. Therefore, the set $J^{\varsigma} \cap \mathcal{N}_{+}$is bounded in $X_{0}$. 


\section{The low initial energy $J\left(u_{0}\right)<d$}

In this section, we consider the global existence and blow-up of weak solution under the condition that $J\left(u_{0}\right)<$ $d$. Specifically, we prove that if $I\left(u_{0}\right)>0$, then problem (1.1) admits a global weak solution and if $I\left(u_{0}\right)<0$, all solutions to problem (1.1) blow up in finite time.

Proof of Theorem 1.1. The proof is divided into three steps.

Step 1: Global existence Noting that $p_{s}^{\star}>2$, we have $X_{0} \subset L^{2}$. Choose sequence $\left\{e_{i}\right\}_{i \in \mathbb{N}} \in C_{0}^{\infty}(\Omega)$, which is an orthogonal basis of $L^{2}(\Omega)$. Consider the following Galerkin approximations:

$$
u^{n}(x, t)=\sum_{i=1}^{n} c_{i}^{n}(t) e_{i}(x),
$$

where functions $c_{k}^{n}(t):[0, T] \rightarrow \mathbb{R}$ satisfy the following system of ordinary differential equations:

$$
\left\{\begin{array}{l}
\left(\frac{\mathrm{d} u^{n}}{\mathrm{~d} t}, e_{j}\right)_{L^{2}(\Omega)}+\left(u^{n}, e_{j}\right)_{X_{0}}=\left(f\left(u^{n}\right), e_{j}\right)_{L^{2}(\Omega)}, \quad j=1,2, \cdots, n \\
u^{n}(x, 0)=\sum_{j=1}^{n}\left(u(x, 0), e_{j}\right)_{L^{2}(\Omega)} e_{j},
\end{array}\right.
$$

with

$$
\begin{gathered}
\left(u^{n}, e_{j}\right)_{X_{0}}=\frac{1}{2} \iint_{Q} \frac{\left|u^{n}(y, t)-u^{n}(x, t)\right|^{p-2}\left(u^{n}(y, t)-u^{n}(x, t)\right)}{|x-y|^{N+p s}}\left(e_{j}(y)-e_{j}(x)\right) \mathrm{d} y \mathrm{~d} x, \\
u^{n}(x, 0) \rightarrow u_{0} \text { in } X_{0}, \quad \text { as } n \rightarrow \infty .
\end{gathered}
$$

It follows from (4.1) that

$$
\begin{aligned}
\frac{\mathrm{d} c_{j}^{n}(t)}{\mathrm{d} t}= & -\frac{1}{2} \iint_{Q} \frac{\left|u^{n}(y, t)-u^{n}(x, t)\right|^{p-2}\left(u^{n}(y, t)-u^{n}(x, t)\right)}{|x-y|^{N+p s}}\left(e_{j}(y)-e_{j}(x)\right) \mathrm{d} y \mathrm{~d} x \\
& +\left(f\left(u^{n}\right), e_{j}\right)_{L^{2}(\Omega)} .
\end{aligned}
$$

Define

$$
\begin{aligned}
F_{j}^{n}\left(\mathbf{c}^{n}(t)\right)= & -\frac{1}{2} \iint_{Q}\left|\sum_{i=1}^{n} c_{i}^{n}(t) e_{i}(y)-\sum_{i=1}^{n} c_{i}^{n}(t) e_{i}(x)\right|^{p-2}\left(\sum_{i=1}^{n} c_{i}^{n}(t) e_{i}(y)-\sum_{i=1}^{n} c_{i}^{n}(t) e_{i}(x)\right) \\
& \times\left(e_{j}(y)-e_{j}(x)\right) \frac{1}{|x-y|^{N+p s}} \mathrm{~d} y \mathrm{~d} x+\left(f\left(\sum_{i=1}^{n} c_{i}^{n}(t) e_{i}(x)\right), e_{j}\right)_{L^{2}(\Omega)},
\end{aligned}
$$

and set

$$
\mathbf{c}^{n}(\cdot)=\left(c_{j}^{n}(\cdot)\right)_{j=1}^{n}, F^{n}(\cdot)=\left(F_{j}^{n}(\cdot)\right)_{j=1}^{n}, \mathbf{c}_{0}^{n}=\left(\left(u_{0}, e_{j}\right)_{L^{2}(\Omega)}\right)_{j=1}^{n} .
$$

Then (4.1) can be rewritten as

$$
\left\{\begin{array}{l}
\frac{\mathrm{dc}^{n}(t)}{\mathrm{d} t}=F^{n}\left(\mathbf{c}^{n}(t)\right), \\
\mathbf{c}^{n}(0)=\mathbf{c}_{0}^{n} .
\end{array}\right.
$$

Next, we aim to use the Peano's theorem to prove the existence of (4.2). Multiplying the first equality of (4.2) by $\mathbf{c}^{n}(t)$, the we get

$$
\begin{aligned}
\frac{1}{2} \frac{\mathrm{d}\left|\mathbf{c}^{n}(t)\right|^{2}}{\mathrm{~d} t}= & -\frac{1}{2} \iint_{Q}\left|\sum_{i=1}^{n} c_{i}^{n}(t) e_{i}(y)-\sum_{i=1}^{n} c_{i}^{n}(t) e_{i}(x)\right|^{p-2}\left(\sum_{i=1}^{n} c_{i}^{n}(t) e_{i}(y)-\sum_{i=1}^{n} c_{i}^{n}(t) e_{i}(x)\right) \\
& \times\left(\sum_{j=1}^{n} c_{j}^{n}(t) e_{j}(y)-\sum_{j=1}^{n} c_{j}^{n}(t) e_{j}(x)\right) \frac{1}{|x-y|^{N+p s}} \mathrm{~d} y \mathrm{~d} x \\
& +\left(f\left(\sum_{i=1}^{n} c_{i}^{n}(t) e_{i}(x)\right), \sum_{j=1}^{n} c_{j}^{n}(t) e_{j}(x)\right)_{L^{2}(\Omega)} .
\end{aligned}
$$


Clearly, the first term on the right side of the above equation is less than 0 , then using Corollary 2.1(1), we get

$$
\begin{aligned}
\frac{1}{2} \frac{\mathrm{d}\left|\mathbf{c}^{n}(t)\right|^{2}}{\mathrm{~d} t} & \leqslant\left(f\left(\sum_{i=1}^{n} c_{i}^{n}(t) e_{i}(x)\right), \sum_{j=1}^{n} c_{j}^{n}(t) e_{j}(x)\right)_{L^{2}(\Omega)} \\
& \leqslant \operatorname{Ar}\left\|\sum_{i=1}^{n} c_{i}^{n}(t) e_{i}(x)\right\|_{L^{r}(\Omega)}^{r} \\
& \leqslant \operatorname{Ar} \sum_{i=1}^{n}\left|c_{i}^{n}(t)\right|^{r} \sum_{i=1}^{n}\left\|e_{i}(x)\right\|_{L^{r}(\Omega)}^{r} \\
& \leqslant \operatorname{Ar}(C(n))^{r} \sum_{i=1}^{n}\left|c_{i}^{n}(t)\right|^{r} \sum_{i=1}^{n}\left\|e_{i}(x)\right\|_{X_{0}}^{r} \\
& \leqslant \operatorname{Ar}(C(n))^{r}\left|\mathbf{c}^{n}(t)\right|^{r},
\end{aligned}
$$

were $C(n)>0$ is a constant depended on $n$. Solving the ordinary differential inequality, we get

$$
\left|\mathbf{c}^{n}(t)\right| \leqslant \frac{1}{\left(\left|\mathbf{c}_{0}^{n}\right|^{1-r / 2}+(2-r) \operatorname{Ar}(C(n))^{r} t\right)^{\frac{2}{r-2}}}, \quad t \in[0, \tilde{T}),
$$

here, $\tilde{T}=\frac{\left|\mathbf{c}_{0}^{n}\right|^{1-r / 2}}{(2-r) \operatorname{Ar}(C(n))^{r}}$. There exists a sufficiently small $\varepsilon>0$ such that $\left|\mathbf{c}^{n}(t)\right| \leqslant C(\tilde{T}-\varepsilon)(C(\tilde{T}-\varepsilon)>0$ is a constant). Furthermore, $F\left(\mathbf{c}^{n}(t)\right)$ with $t \in[0, \tilde{T}-\varepsilon]$ is bounded(denote by $G_{0}$ the boundedness). Denote

$$
T_{0}=0, \quad E:=\left\{\left(t, \mathbf{c}^{n}(t)\right) \in \mathbb{R} \times \mathbb{R}^{n}|| t-T_{0}|\leqslant \tilde{T},| \mathbf{c}^{n}(t)-\mathbf{c}_{0}^{n} \mid \leqslant C(\tilde{T}-\varepsilon)\right\}, \quad \forall t \in[0, \tilde{T}) .
$$

Peano's theorem implies that there exists a solutions $\mathbf{c}^{n}(t)$ of (4.2) on $\left[0, T_{1}\right]$, where

$$
T_{1}=\min \left\{\tilde{T}-\varepsilon, \frac{\mathbf{c}_{0}^{n}}{G_{0}}\right\}
$$

For $t \in\left[0, T_{1}\right]$, the following (4.6) still holds, then we have

$$
\left|c_{j}^{n}(t)\right|^{2} \leqslant \sum_{j=1}^{n}\left|c_{j}^{n}(t)\right|^{2}=\sum_{j=1}^{n}\left|c_{j}^{n}(t)\right|^{2} \int_{\Omega}\left|e_{j}\right|^{2} \mathrm{~d} x=\left\|u^{n}\right\|_{L^{2}(\Omega)}^{2} \leqslant C_{\star}^{2}\left\|u^{n}\right\|_{X_{0}}^{2}<C_{\star}^{2}\left(\frac{d p(q+1)}{q+1-p}\right)^{\frac{2}{p}} .
$$

Furthermore, $F\left(\mathbf{c}^{n}(t)\right)$ is bounded. Denote by $T_{1}$ the new initial point, Peano's theorem implies that there exists a global solution $\mathbf{c}^{n}(t)$ of the ordinary differential equation (4.2) by repeating the similar argument. we get

Multiplying (4.1) by $\frac{\mathrm{d} c_{j}^{n}(t)}{\mathrm{d} t}$, and summing for $j$ from 0 to $n$, then integrating with respect to $t$ from 0 to $t$,

$$
\int_{0}^{t}\left\|u_{\tau}^{n}\right\|_{L^{2}(\Omega)}^{2} \mathrm{~d} \tau+J\left(u^{n}\right)=J\left(u^{n}(0)\right)
$$

It follows from $u^{n}(x, 0) \rightarrow u_{0}(x)$ in $X_{0}$ that

$$
J\left(u^{n}(x, 0)\right) \rightarrow J\left(u_{0}(x)\right)<d \text { and } I\left(u^{n}(x, 0)\right) \rightarrow I\left(u_{0}(x)\right)>0, \quad \text { as } n \rightarrow \infty .
$$

Therefore, for sufficiently large $n$, we get

$$
\int_{0}^{t}\left\|u_{\tau}^{n}\right\|_{L^{2}(\Omega)}^{2} \mathrm{~d} \tau+J\left(u^{n}\right)=J\left(u^{n}(0)\right)<d \text { and } I\left(u^{n}(x, 0)\right)>0,
$$

which implies that $u^{n}(x, 0) \in W$.

Next, we prove $u^{n}(x, t) \in W$ for sufficiently large $n$. Otherwise, there exists a $t_{0} \in(0, \infty)$ such that $u^{n}\left(x, t_{0}\right) \in \partial W$, that is

$$
I\left(u^{n}\left(x, t_{0}\right)\right)=0,\|u\|_{X_{0}} \neq 0 \text { or } J\left(u^{n}\left(x, t_{0}\right)\right)=d .
$$


Clearly, $J\left(u^{n}\left(x, t_{0}\right)\right) \neq d$ from (4.4). If $I\left(u^{n}\left(x, t_{0}\right)\right)=0,\|u\|_{X_{0}} \neq 0$, then $J\left(u^{n}\left(x, t_{0}\right)\right) \geqslant d$ by the definition of $d$, which contradicts (4.4). Therefore, $u^{n}(x, t) \in W$ and $I\left(u^{n}\right)>0$. Combining with condition (c), we have

$$
J\left(u^{n}\right) \geqslant \frac{1}{p}\left\|u^{n}\right\|_{X_{0}}^{p}-\frac{1}{q+1} \int_{\Omega} u^{n} f\left(u^{n}\right) \mathrm{d} x=\frac{q+1-p}{p(q+1)}\left\|u^{n}\right\|_{X_{0}}^{p}+\frac{1}{q+1} I\left(u^{n}\right) .
$$

From (4.4), we get

$$
\int_{0}^{t}\left\|u_{\tau}^{n}\right\|_{L^{2}(\Omega)}^{2} \mathrm{~d} \tau+\frac{q+1-p}{p(q+1)}\left\|u^{n}\right\|_{X_{0}}^{p}+\frac{1}{q+1} I\left(u^{n}\right)<d,
$$

which implies

$$
\begin{gathered}
\left\|u^{n}\right\|_{X_{0}}^{p}<\frac{d p(q+1)}{q+1-p}, \\
\int_{0}^{t}\left\|u_{\tau}^{n}\right\|_{L^{2}(\Omega)}^{2} \mathrm{~d} \tau<d .
\end{gathered}
$$

Furthermore, Corollary 2.1(1) and the embedding from $X_{0}$ into $L^{r}(\Omega)$ imply

$$
\left\|f\left(u^{n}\right)\right\|_{L^{\frac{r}{r-1}(\Omega)}} \leqslant \operatorname{Ar}\left\|u^{n}\right\|_{L^{r}(\Omega)}^{r-1} \leqslant \operatorname{Ar}\left(C_{\star}\right)^{r-1}\left\|u^{n}\right\|_{X_{0}}^{r-1}<\operatorname{Ar}\left(C_{\star}\right)^{r-1}\left(\frac{d p(q+1)}{q+1-p}\right)^{\frac{r-1}{p}} .
$$

Therefore, there exists a $u$ and a subsequence of $\left\{u^{n}\right\}_{n \in \mathbb{N}}$ (still denoted by $\left\{u^{n}\right\}_{n \in \mathbb{N}}$ ) such that as $n \rightarrow \infty$,

$$
\begin{aligned}
u^{n} \stackrel{\star}{\rightarrow} u & \text { in } L^{\infty}\left(0, \infty ; X_{0}\right) ; \\
u_{t}^{n} \rightarrow u_{t} & \text { in } L^{2}\left(0, \infty ; L^{2}(\Omega)\right) ; \\
f\left(u^{n}\right) \stackrel{\star}{\rightarrow} \xi & \text { in } L^{\infty}\left(0, \infty ; L^{\frac{r}{r-1}}(\Omega)\right) .
\end{aligned}
$$

Since the embedding from $X_{0}$ into $L^{r}(\Omega)$ is compact and the embedding from $L^{r}(\Omega)$ into $L^{2}(\Omega)$ is continuous, from Simon's theorem [29] we get

$$
u^{n} \rightarrow u \quad \text { in } C\left([0, \infty] ; L^{r}(\Omega)\right), \quad \text { as } n \rightarrow \infty .
$$

Therefore, $\xi=f(u)$.

Next, we show that the function $u$ is a weak solution of the problem (1.1). Choose $v \subset C^{1}\left([0, \infty] ; C_{0}^{\infty}(\Omega)\right)$ with the following form

$$
v=\sum_{j=1}^{k} l_{j}(t) e_{j}
$$

where $l_{j}(t) \in C^{1}([0, \infty])$ with $j=1,2, \cdots, k(k \leqslant n)$. Multiplying the first equality of (4.1) by $l_{j}(t)$ summing for $j$ from 1 to $n$, integrating with respect to $t$ from 0 to $T$, we get

$$
\int_{0}^{T}\left(u_{t}^{n}, v\right)_{L^{2}(\Omega)} \mathrm{d} t+\int_{0}^{T}\left(u^{n}, v\right)_{X_{0}} \mathrm{~d} t=\int_{0}^{T}\left(f\left(u^{n}\right), v\right)_{L^{2}(\Omega)} \mathrm{d} t .
$$

Letting $n \rightarrow \infty$, combining with the theory of monotone operators as in [24], we get

$$
\int_{0}^{T}\left(u_{t}, v\right)_{L^{2}(\Omega)} \mathrm{d} t+\int_{0}^{T}(u, v)_{X_{0}} \mathrm{~d} t=\int_{0}^{T}(f(u), v)_{L^{2}(\Omega)} \mathrm{d} t .
$$

Since $C^{1}\left([0, \infty] ; C_{0}^{\infty}(\Omega)\right)$ is dense in $L^{2}\left(0, \infty ; X_{0}\right)$, the identity in (4.10) holds for $v \in L^{2}\left(0, \infty ; X_{0}\right)$. Moreover, by the arbitrariness of $T>0$, we get

$$
\left(u_{t}, \varphi\right)_{L^{2}(\Omega)}+(u, \varphi)_{X_{0}}=(f(u), \varphi)_{L^{2}(\Omega)}, \quad \varphi \in X_{0} \text {, a.e. } t>0 .
$$


By (4.9) and $u^{n}(x, 0) \rightarrow u_{0}(x)$ in $X_{0}$, then $u(x, 0)=u_{0}(x)$. Assuming that $u$ is sufficiently smooth such that $u_{t} \in L^{2}\left(0, \infty ; X_{0}\right)$, taking $v=u_{t}$ in (4.10), then (3.5) the holds. Since $L^{2}\left(0, \infty ; X_{0}\right)$ is dense in $L^{2}\left(0, \infty ; L^{2}(\Omega)\right)$, then (3.5) holds for weak solutions of problem (1.1).

Step 2: Uniqueness of bounded weak solution Assuming both $u, v$ are two bounded weak solutions for problem (1.1). Then by the definition of weak solution, for $\varphi \in X_{0}$, we get

$$
\begin{gathered}
\left(u_{t}, \varphi\right)_{L^{2}(\Omega)}+(u, \varphi)_{X_{0}}=(f(u), \varphi)_{L^{2}(\Omega)} ; \\
\left(v_{t}, \varphi\right)_{L^{2}(\Omega)}+(v, \varphi)_{X_{0}}=(f(v), \varphi)_{L^{2}(\Omega)} .
\end{gathered}
$$

Subtracting the above two equalities, taking $\varphi=u-v \in X_{0}$, and then integrating for $t$ from 0 to $t$, we get

$$
\begin{aligned}
& \int_{0}^{t} \iint_{Q}\left[|u(y, t)-u(x, t)|^{p-2}(u(y, t)-u(x, t))-|v(y, t)-v(x, t)|^{p-2}(v(y, t)-v(x, t)]\right. \\
& \quad \times[u(y, t)-u(x, t)-v(y, t)+v(x, t)] \frac{1}{|x-y|^{N+p s}} \mathrm{~d} y \mathrm{~d} x \mathrm{~d} t+\int_{0}^{t} \int_{\Omega} \varphi_{t} \varphi \mathrm{d} x \mathrm{~d} t \\
& =\int_{0}^{t} \int_{\Omega}(f(u)-f(v)) \varphi \mathrm{d} x \mathrm{~d} t .
\end{aligned}
$$

Clearly, the fist term on the left side of the above equality is non-negative. By the continuity of $f$, boundedness of $u, v$ and Cauchy-Schwarz inequality we get

$$
\int_{0}^{t} \int_{\Omega} \varphi_{t} \varphi \mathrm{d} x \mathrm{~d} t \leqslant \int_{0}^{t} \int_{\Omega}(f(u)-f(v)) \varphi \mathrm{d} x \mathrm{~d} t \leqslant C \int_{0}^{t} \int_{\Omega} \varphi^{2} \mathrm{~d} x \mathrm{~d} t,
$$

here $C>0$ depends on the bound of $u, v$. Furthermore,

$$
\int_{\Omega} \varphi^{2} \mathrm{~d} x \leqslant C \int_{0}^{t} \int_{\Omega} \varphi^{2} \mathrm{~d} x \mathrm{~d} t
$$

by $\varphi(x, 0)=0$. Gronwall's inequality implies

$$
\int_{\Omega} \varphi^{2} \mathrm{~d} x=0
$$

Thus $\varphi=0$ a.e. in $\Omega \times(0, \infty)$.

Step 3: Upper estimate Taking $\varphi=u$ in (3.4), we obtain

$$
\frac{1}{2} \frac{\mathrm{d}}{\mathrm{d} t}\|u\|_{L^{2}(\Omega)}^{2}=-I(u) .
$$

From Lemma 3.2 and Lemma 3.7, we know that $u(x, t) \in W_{\delta}$ for $\delta_{1}<\delta<\delta_{2}$ and $0<t<\infty$ under the condition $J\left(u_{0}\right)<d$ and $I\left(u_{0}\right)>0$. Thus Lemma 3.6 implies $I_{\delta_{1}}(u) \geqslant 0$ for $0<t<\infty$. Therefore, the embedding from $X_{0}$ into $L^{2}(\Omega)$ implies that

$$
\frac{1}{2} \frac{\mathrm{d}}{\mathrm{d} t}\|u\|_{L^{2}(\Omega)}^{2}=-I(u)=\left(\delta_{1}-1\right)\|u\|_{X_{0}}^{p}-I_{\delta_{1}}(u) \leqslant\left(\delta_{1}-1\right) C_{\star}^{-p}\|u\|_{L^{2}(\Omega)}^{p} .
$$

Thus for $p<2$, we get

$$
\|u\|_{L^{2}(\Omega)}^{2} \leqslant\left(\left\|u_{0}\right\|_{L^{2}(\Omega)}^{2-p}-(2-p)\left(1-\delta_{1}\right) C_{\star}^{-p} t\right)_{+}^{\frac{2}{2-p}}, \quad \forall 0 \leqslant t<\infty,
$$

where

$$
\left(\left\|u_{0}\right\|_{L^{2}(\Omega)}^{2-p}-(2-p)\left(1-\delta_{1}\right) C_{\star}^{-p} t\right)_{+}=\max \left\{\left\|u_{0}\right\|_{L^{2}(\Omega)}^{2-p}-(2-p)\left(1-\delta_{1}\right) C_{\star}^{-p}, 0\right\} .
$$


This means that the solution $u$ vanishes at a finite time $t^{\star}=\frac{\left\|u_{0}\right\|_{L^{2}(\Omega)}^{2-p}}{(2-p)\left(1-\delta_{1}\right) C_{\star}^{-p}}$.

For $p=2$, we get

$$
\|u\|_{L^{2}(\Omega)}^{2} \leqslant\left\|u_{0}\right\|_{L^{2}(\Omega)}^{2} e^{-2\left(1-\delta_{1}\right) C_{*}^{-2} t}, \quad \forall 0 \leqslant t<\infty .
$$

For $p>2$, we get

$$
\|u\|_{L^{2}(\Omega)}^{2} \leqslant\left(\frac{1}{(2-p)\left(\delta_{1}-1\right) C_{\star}^{-p} t+\left\|u_{0}\right\|_{L^{2}(\Omega)}^{2-p}}\right)^{\frac{2}{p-2}}, \quad \forall 0 \leqslant t<\infty .
$$

Next, we are concerned with blow-up in finite time.

Proof of Theorem 1.2. Assume that $u$ is a global weak solution of problem (1.1) with $J\left(u_{0}\right)<d, I\left(u_{0}\right)<0$, and define

$$
M(t)=\int_{0}^{t}\|u\|_{L^{2}(\Omega)}^{2} \mathrm{~d} \tau, \quad \forall t \geqslant 0
$$

Then

$$
M^{\prime}(t)=\|u\|_{L^{2}(\Omega)}^{2},
$$

and

$$
M^{\prime \prime}(t)=2\left(u_{t}, u\right)_{L^{2}(\Omega)}=-2 I(u) .
$$

Using condition $(c)$, we compute to obtain

$$
\begin{aligned}
J(u) & \geqslant \frac{1}{p}\|u\|_{X_{0}}^{p}-\frac{1}{q+1} \int_{\Omega} u f(u) \mathrm{d} x \\
& =\frac{q+1-p}{p(q+1)}\|u\|_{X_{0}}^{p}+\frac{1}{q+1} I(u) .
\end{aligned}
$$

Using (3.5), (4.13) and (4.14), we get

$$
\begin{aligned}
M^{\prime \prime}(t) & \geqslant \frac{2(q+1-p)}{p}\|u\|_{X_{0}}^{p}-2(q+1) J(u) \\
& \geqslant \frac{2(q+1-p)}{p C_{\star}^{p}}\|u\|_{L^{2}(\Omega)}^{p}+2(q+1) \int_{0}^{t}\left\|u_{\tau}\right\|_{L^{2}(\Omega)}^{2} \mathrm{~d} \tau-2(q+1) J\left(u_{0}\right) \\
& =\frac{2(q+1-p)}{p C_{\star}^{p}}\left(M^{\prime}(t)\right)^{\frac{p}{2}}+2(q+1) \int_{0}^{t}\left\|u_{\tau}\right\|_{L^{2}(\Omega)}^{2} \mathrm{~d} \tau-2(q+1) J\left(u_{0}\right) .
\end{aligned}
$$

Noticing that

$$
\left(M^{\prime}(t)\right)^{2}=4\left(\int_{0}^{t} \int_{\Omega} u_{\tau} u \mathrm{~d} x \mathrm{~d} \tau\right)^{2}+2\left\|u_{0}\right\|_{L^{2}(\Omega)}^{2} M^{\prime}(t)-\left\|u_{0}\right\|_{L^{2}(\Omega)}^{4}
$$

then we have

$$
\begin{aligned}
M^{\prime \prime}(t) M(t)-\frac{q+1}{2}\left(M^{\prime}(t)\right)^{2} \geqslant & \frac{2(q+1-p)}{p C_{\star}^{p}}\left(M^{\prime}(t)\right)^{\frac{p}{2}} M(t)-2 M(t)(q+1) J\left(u_{0}\right) \\
& +2(q+1) \int_{0}^{t}\|u\|_{L^{2}(\Omega)}^{2} \mathrm{~d} \tau \int_{0}^{t}\left\|u_{\tau}\right\|_{L^{2}(\Omega)}^{2} \mathrm{~d} \tau+\frac{q+1}{2}\left\|u_{0}\right\|_{L^{2}(\Omega)}^{4} \\
& -2(q+1)\left(\int_{0}^{t} \int_{\Omega} u_{\tau} u \mathrm{~d} x \mathrm{~d} \tau\right)^{2}-(q+1)\left\|u_{0}\right\|_{L^{2}(\Omega)}^{2} M^{\prime}(t) .
\end{aligned}
$$


By Cauchy-Schwarz inequality

$$
\left(\int_{0}^{t} \int_{\Omega} u_{\tau} u \mathrm{~d} x \mathrm{~d} \tau\right)^{2} \leqslant \int_{0}^{t}\|u\|_{L^{2}(\Omega)}^{2} \mathrm{~d} \tau \int_{0}^{t}\left\|u_{\tau}\right\|_{L^{2}(\Omega)}^{2} \mathrm{~d} \tau
$$

we get

$$
\begin{aligned}
M^{\prime \prime}(t) M(t)-\frac{q+1}{2}\left(M^{\prime}(t)\right)^{2} \geqslant & \frac{2(q+1-p)}{p C_{\star}^{p}}\left(M^{\prime}(t)\right)^{\frac{p}{2}} M(t)-2 M(t)(q+1) J\left(u_{0}\right) \\
& -(q+1)\left\|u_{0}\right\|_{L^{2}(\Omega)}^{2} M^{\prime}(t) .
\end{aligned}
$$

Now, we discuss the following two cases:

Case 1: $J\left(u_{0}\right) \leqslant 0$

For $J\left(u_{0}\right) \leqslant 0$, then (4.15) implies

$$
M^{\prime \prime}(t) M(t)-\frac{q+1}{2}\left(M^{\prime}(t)\right)^{2} \geqslant \frac{2(q+1-p)}{p C_{*}^{p}}\left(M^{\prime}(t)\right)^{\frac{p}{2}} M(t)-(q+1)\left\|u_{0}\right\|_{L^{2}(\Omega)}^{2} M^{\prime}(t) .
$$

Now we show that $I(u)<0$ for $t>0$. Otherwise, there exists a $t_{0}>0$ such that $I\left(u\left(t_{0}\right)\right)=0$ and $I(u)<0$ for $0 \leqslant t<t_{0}$. From Lemma 3.4(2)(3), $\|u\|_{X_{0}}>y(1)$ for $0 \leqslant t<t_{0}$, and $\left\|u\left(t_{0}\right)\right\|_{X_{0}} \geqslant y(1)$. Therefore, $J\left(u\left(t_{0}\right)\right) \geqslant d$, which contradicts (3.5). Then (4.13) implies $M^{\prime \prime}(t)>0$ for $t \geqslant 0$. Since $M^{\prime}(0)=\left\|u_{0}\right\|_{L^{2}(\Omega)}^{2} \geqslant 0$, there exists a $t_{0}>0$ such that $M^{\prime}\left(t_{0}\right)>0$. Thus, we get

$$
M(t)=M\left(t_{0}\right)+\int_{t_{0}}^{t} M^{\prime}(\tau) \mathrm{d} \tau \geqslant M^{\prime}\left(t_{0}\right)\left(t-t_{0}\right)
$$

Then for any

$$
t \geqslant t^{\star}:=\max \left\{t_{0}, \frac{(q+1)\left\|u_{0}\right\|_{L^{2}(\Omega)}^{2}+\frac{2(q+1-p)}{p c_{\star}^{p}}\left(M^{\prime}\left(t_{0}\right)\right)^{\frac{p}{2}-1} M^{\prime}\left(t_{0}\right) t_{0}}{\frac{2(q+1-p)}{p C_{\star}^{p}}\left(M^{\prime}\left(t_{0}\right)\right)^{\frac{p}{2}-1} M^{\prime}\left(t_{0}\right)}\right\},
$$

we have

$$
\begin{aligned}
M^{\prime \prime}(t) M(t)-\frac{q+1}{2}\left(M^{\prime}(t)\right)^{2} \geqslant & M^{\prime}(t)\left(\frac{2(q+1-p)}{p C_{\star}^{p}}\left(M^{\prime}(t)\right)^{\frac{p}{2}-1} M(t)-(q+1)\left\|u_{0}\right\|_{L^{2}(\Omega)}^{2}\right) \\
\geqslant & M^{\prime}(t)\left(\frac{2(q+1-p)}{p C_{\star}^{p}}\left(M^{\prime}\left(t_{0}\right)\right)^{\frac{p}{2}-1} M^{\prime}\left(t_{0}\right)\left(t-t_{0}\right)\right. \\
& \left.-(q+1)\left\|u_{0}\right\|_{L^{2}(\Omega)}^{2}\right) \\
\geqslant & 0 .
\end{aligned}
$$

Case 2: $0<J\left(u_{0}\right)<d$

By Lemma 3.7(2), we have $u(x, t) \in V_{\delta}$ for $\delta_{1}<\delta<\delta_{2}$ and $0<t<\infty$. Hence, Lemma 3.6 implies $I_{\delta_{2}}(u) \leqslant 0$. Furthermore, Lemma 3.4 implies $\|u\|_{X_{0}} \geqslant y\left(\delta_{2}\right)$ for $t \geqslant 0$. It follows from (4.13) that for $t \geqslant 0$, we get

$$
M^{\prime \prime}(t)=-2 I(u)=2\left(\delta_{2}-1\right)\|u\|_{X_{0}}^{p}-2 I_{\delta_{2}}(u) \geqslant C_{\star \star}:=2\left(\delta_{2}-1\right)\left(y\left(\delta_{2}\right)\right)^{p},
$$

which implies

$$
\begin{aligned}
M^{\prime}(t) & \geqslant C_{\star \star} t ; \\
M(t) & \geqslant \frac{C_{\star \star}}{2} t^{2},
\end{aligned}
$$

and $M^{\prime}(t)>0, M(t)>0$ for any $t>0$. Considering (4.15), for any

$$
t \geqslant t^{\star}:=\max \left\{\left(\frac{2(q+1) J\left(u_{0}\right)}{\frac{q+1-p}{p C_{\star}^{p}} C_{\star \star}^{\frac{p}{2}}}\right)^{\frac{2}{p}},\left(\frac{(q+1)\left\|u_{0}\right\|_{L^{2}(\Omega)}^{2}}{\frac{q+1-p}{p C_{\star}^{p}} \frac{C_{* \star}^{2}}{2}}\right)^{\frac{2}{p+2}}\right\},
$$


we have

$$
\begin{aligned}
M^{\prime \prime}(t) M(t)-\frac{q+1}{2}\left(M^{\prime}(t)\right)^{2} \geqslant & M(t)\left(\frac{q+1-p}{p C_{\star}^{p}}\left(M^{\prime}(t)\right)^{\frac{p}{2}}-2(q+1) J\left(u_{0}\right)\right) \\
& +M^{\prime}(t)\left(\frac{q+1-p}{p C_{\star}^{p}}\left(M^{\prime}(t)\right)^{\frac{p}{2}-1} M(t)-(q+1)\left\|u_{0}\right\|_{L^{2}(\Omega)}^{2}\right) \\
\geqslant & 0 .
\end{aligned}
$$

From the above discussion, there exists $t^{\star}>0$ such that

$$
M^{\prime \prime}(t) M(t)-\frac{q+1}{2}\left(M^{\prime}(t)\right)^{2} \geq 0, \quad t \geq t^{\star} .
$$

Following the concavity method introduced by Levine [15], we can get the estimate of $T^{\star}$. For more details, one can also refer to [13, Lemma 1.1] and [16, Lemma 2.1]. In fact, by a direct computation, we see that

$$
\left(M^{-\theta}(t)\right)^{\prime \prime}=\theta M^{-\theta-2}(t)\left((1+\theta)\left(M^{\prime}(t)\right)^{2}-M(t) M^{\prime \prime}(t)\right) \leq 0, \quad t \geq t^{\star},
$$

where $\theta=\frac{q-1}{2}$. Since a concave function must always lie below any tangent line, we can obtain

$$
0<M^{-\theta}(t) \leq M^{-\theta}\left(t^{\star}\right)+\left(M^{-\theta}\left(t^{\star}\right)\right)^{\prime} t, \quad t \geq t^{\star} .
$$

Then from the fact that $M\left(t^{\star}\right)>0$ and $M^{\prime}\left(t^{\star}\right)>0$, we get that $T^{\star} \leqslant t^{\star}+\frac{M\left(t^{\star}\right)}{\frac{q-1}{2} M^{\prime}\left(t^{\star}\right)}$ and $M(t) \rightarrow \infty$ as $t \rightarrow T^{\star}$, which contradicts our assumption that $u$ is a global weak solution of problem (1.1). The proof is completed.

\section{The critical initial energy $J\left(u_{0}\right)=d$}

For critical initial energy $J\left(u_{0}\right)=d$, in this section, we show that if $I\left(u_{0}\right) \geqslant 0$, then problem (1.1) admits a global weak solution and if $I\left(u_{0}\right)<0$, all solutions to problem (1.1) blow up in finite time.

Proof of Theorem 1.3. Let $\lambda_{k}=1-\frac{1}{k}, k=1,2, \cdots$. Consider the following initial value problem:

$$
\begin{cases}u_{t}(x, t)+(-\Delta)_{p}^{s} u(x, t)=f(u(x, t)), & (x, t) \in \Omega \times(0, T), \\ u(x, t)=0, & (x, t) \in \mathcal{C} \Omega \times(0, T), \\ u(x, 0)=\lambda_{k} u_{0}(x):=u_{0}^{k}, & x \in \Omega .\end{cases}
$$

Noticing that $I\left(u_{0}\right) \geqslant 0$, by Lemma 3.3(3) we can deduce that there exists a unique $\lambda^{\star}=\lambda^{\star}\left(u_{0}\right) \geqslant 1$ such that $I\left(\lambda^{\star} u_{0}\right)=0$. Then from $\lambda_{k}<1 \leqslant \lambda^{\star}$ and Lemma 3.3(2)(3), we get $I\left(u_{0}^{k}\right)=I\left(\lambda_{k} u_{0}\right)>0$ and $J\left(u_{0}^{k}\right)=$ $J\left(\lambda_{k} u_{0}\right)<J\left(u_{0}\right)=d$. In view of Theorem 1.1, for each $k$, then problem (5.1) admits a global weak solution $u^{k} \in L^{\infty}\left(0, \infty ; X_{0}\right)$ with $u_{t}^{k} \in L^{2}\left(0, \infty ; L^{2}(\Omega)\right)$ and $u^{k} \in W$ satisfying

$$
\int_{0}^{t}\left\|u_{\tau}^{k}\right\|_{L^{2}(\Omega)}^{2} \mathrm{~d} \tau+J\left(u^{k}\right)=J\left(u_{0}^{k}\right)<d .
$$

Applying the similar argument in Theorem 1.1, there exists a subsequence $\left\{u^{k}\right\}_{k \in \mathbb{N}}$ which converges to a function $u$ and $u$ is a weak solution of problem (1.1) with $I(u) \geqslant 0$ and $J(u) \leqslant d$ for $0 \leqslant t<\infty$. The proof of uniqueness for bounded weak solution is the same as that in Theorem 1.1.

Let us consider $\|u\|_{L^{2}(\Omega)}^{2}$. Firstly, assume that $I(u)>0$ for $0<t<\infty$. Then (4.11) implies $u_{t} \neq 0$. Therefore, by Lemma 3.2 and (3.5) for any $t_{0}>0$ we have

$$
0<J\left(u\left(t_{0}\right)\right)=d-\int_{0}^{t_{0}}\left\|u_{\tau}\right\|_{L^{2}(\Omega)}^{2} \mathrm{~d} \tau<d .
$$


Taking $t=t_{0}$ as the initial time, from Lemma 3.7, we know that $u(x, t) \in W_{\delta}$ for $\delta_{1}<\delta<\delta_{2}$ and $t_{0}<t<\infty$ under the condition $J\left(u\left(t_{0}\right)\right)<d$ and $I\left(u\left(t_{0}\right)\right)>0$, where $\delta_{1}<1<\delta_{2}$ are the two roots of $d(\delta)=J\left(u\left(t_{0}\right)\right)$. Thus Lemma 3.6 implies $I_{\delta_{1}}(u) \geqslant 0$ for $t_{0}<t<\infty$. Therefore, the embedding from $X_{0}$ into $L^{2}(\Omega)$ implies

$$
\frac{1}{2} \frac{\mathrm{d}}{\mathrm{d} t}\|u\|_{L^{2}(\Omega)}^{2}=-I(u)=\left(\delta_{1}-1\right)\|u\|_{X_{0}}^{p}-I_{\delta_{1}}(u) \leqslant\left(\delta_{1}-1\right) C_{\star}^{-p}\|u\|_{L^{2}(\Omega)}^{p} .
$$

Thus for $p<2$, we get

$$
\|u\|_{L^{2}(\Omega)}^{2} \leqslant\left(\left\|u\left(t_{0}\right)\right\|_{L^{2}(\Omega)}^{2-p}-(2-p)\left(1-\delta_{1}\right) C_{*}^{-p} t\right)_{+}^{\frac{2}{2-p}}, \quad \forall 0<t_{0} \leqslant t<\infty
$$

where

$$
\left(\left\|u\left(t_{0}\right)\right\|_{L^{2}(\Omega)}^{2-p}-(2-p)\left(1-\delta_{1}\right) C_{\star}^{-p} t\right)_{+}=\max \left\{\left\|u\left(t_{0}\right)\right\|_{L^{2}(\Omega)}^{2-p}-(2-p)\left(1-\delta_{1}\right) C_{\star}^{-p}, 0\right\} .
$$

This means that the solution $u$ vanishes at a finite time $T^{\star}=\frac{\left\|u\left(t_{0}\right)\right\|_{L^{2}(\Omega)}^{2-p}}{(2-p)\left(1-\delta_{1}\right) C_{*}^{-p}}$.

For $p=2$, we get

$$
\|u\|_{L^{2}(\Omega)}^{2} \leqslant\left\|u\left(t_{0}\right)\right\|_{L^{2}(\Omega)}^{2} e^{-2\left(1-\delta_{1}\right) C_{*}^{-2} t}, \quad \forall 0<t_{0} \leqslant t<\infty .
$$

For $p>2$, we get

$$
\|u\|_{L^{2}(\Omega)}^{2} \leqslant\left(\frac{1}{(2-p)\left(\delta_{1}-1\right) C_{\star}^{-p} t+\left\|u\left(t_{0}\right)\right\|_{L^{2}(\Omega)}^{2-p}}\right)^{\frac{2}{p-2}}, \quad \forall 0<t_{0} \leqslant t<\infty .
$$

Secondly, assume that $I(u)>0$ for $0<t<t^{\star}$ and $I\left(u\left(t^{\star}\right)\right)=0$. Then (4.11) implies $u_{t} \neq 0$ for $0<t<t^{\star}$. Therefore, by (3.5) we have

$$
J\left(u\left(t^{\star}\right)\right)=d-\int_{0}^{t^{\star}}\left\|u_{\tau}\right\|_{L^{2}(\Omega)}^{2} \mathrm{~d} \tau<d .
$$

By the definition of $d$, we easily know $\left\|u\left(t^{\star}\right)\right\|_{X_{0}}=0$, which implies $u\left(t^{\star}\right)=0$. Define $u(t) \equiv 0$ for $t \geqslant t^{\star}$. Then, such a weak solution $u(x, t)$ vanishes in finite time $t^{\star}$.

Next, we are concerned with blow-up in finite time.

Proof of Theorem 1.4. By the same argument in (4.15) of Theorem 1.2, we get

$$
\begin{aligned}
M^{\prime \prime}(t) M(t)-\frac{q+1}{2}\left(M^{\prime}(t)\right)^{2} \geqslant & \frac{2(q+1-p)}{p C_{\star}^{p}}\left(M^{\prime}(t)\right)^{\frac{p}{2}} M(t)-2 M(t)(q+1) d \\
& -(q+1)\left\|u_{0}\right\|_{L^{2}(\Omega)}^{2} M^{\prime}(t) .
\end{aligned}
$$

Since $J\left(u_{0}\right)=d, I\left(u_{0}\right)<0$, by the continuity of $J(u)$ and $I(u)$ with respect to $t$, there exists a $t_{0}$ such that $J(u(x, t))>0$ and $I(u(x, t))<0$ for $0<t \leqslant t_{0}$. We have $u_{t} \neq 0$ from (4.11) for $0<t \leqslant t_{0}$, moreover $\int_{0}^{t_{0}}\left\|u_{\tau}\right\|_{L^{2}(\Omega)}^{2} \mathrm{~d} \tau>0$. Then from (3.5)

$$
0<J\left(u\left(t_{0}\right)\right)=d-\int_{0}^{t_{0}}\left\|u_{\tau}\right\|_{L^{2}(\Omega)}^{2} \mathrm{~d} \tau:=d_{1}<d .
$$

Taking $t=t_{0}$ as the initial time and by Lemma 3.7(2), we get $u(x, t) \in V_{\delta}$ for $\delta_{1}<\delta<\delta_{2}$ and $t_{0}<t$, where $\delta_{1}<1<\delta_{2}, \delta_{1}, \delta_{2}$ satisfy the equation $d(\delta)=d_{1}$. Therefore, $I_{\delta}(u)<0$ and $\|u\|_{X_{0}}>y(\delta)$ for $\delta_{1}<\delta<\delta_{2}$ and $t_{0}<t$ by Lemma 3.4(2). Furthermore, combining with Lemma 3.6, we get $I_{\delta_{2}}(u) \leqslant 0$ and $\|u\|_{X_{0}} \geqslant y\left(\delta_{2}\right)$ for $t_{0}<t$. The rest of this proof is the same as that one of Case 2 in Theorem 1.2., here, we omit the process. 


\section{The high initial energy $J\left(u_{0}\right)>d$}

In this section, we give some sufficient conditions for global existence of weak solutions and blow-up in finite time regarding the high initial energy. Before proving our results, we need the following lemma.

Lemma 6.1. For any $\varsigma>d, \lambda_{\varsigma}$ and $\Lambda_{\varsigma}$ satisfy

$$
0<\lambda_{\varsigma} \leqslant \Lambda_{\varsigma}<+\infty .
$$

Proof. If $u \in \mathcal{N}^{\varsigma}$, then it follows from Corollary 2.1(1) and Hölder’s inequality that

$$
\|u\|_{X_{0}}^{p}=\int_{\Omega} u f(u) \mathrm{d} x \leqslant A r\|u\|_{L^{r}(\Omega)}^{r} \leqslant A r\|u\|_{L^{p_{s}^{*}(\Omega)}}^{\alpha r}\|u\|_{L^{2}(\Omega)}^{(1-\alpha) r} \leqslant \operatorname{Ar} C_{*}^{\alpha r}\|u\|_{X_{0}}^{\alpha r}\|u\|_{L^{2}(\Omega)}^{(1-\alpha) r},
$$

where $\alpha \in(0,1)$ since $r<p_{s}^{\star}$. (6.2) can be written as

$$
\|u\|_{L^{2}(\Omega)}^{(1-\alpha) r} \geqslant \frac{1}{\operatorname{ArC}_{\star}^{\alpha r}}\|u\|_{X_{0}}^{p-\alpha r} .
$$

Clearly, the right term of (6.3) is bounded and away from 0 by Lemma 3.8(1) and the definition of $\mathcal{N}^{\varsigma}$. Therefore, we get $\lambda_{\varsigma}>0$ by the definition of $\lambda_{\varsigma}$. Embedding $X_{0}$ into $L^{2}(\Omega)$, we get $\|u\|_{L^{2}(\Omega)}^{2} \leqslant C_{\star}^{2}\|u\|_{X_{0}}^{2}$. Combining with the definition of $\mathcal{N}^{\varsigma}$, it is obvious that $\Lambda_{\varsigma}<+\infty$.

Proof of Theorem 1.5. We denote by $T\left(u_{0}\right)$ the maximal existence time of the solutions for problem (1.1). If there exists a global solution, that is, $T\left(u_{0}\right)=\infty$, we denote by

$$
\omega\left(u_{0}\right)=\bigcap_{t \geqslant 0} \overline{\{u(\iota): \iota \geqslant t\}}^{X_{0}}
$$

the $\omega$-limit of $u_{0} \in X_{0}$.

(1) If $u_{0} \in \mathcal{N}_{+}$and $\left\|u_{0}\right\|_{L^{2}(\Omega)} \leqslant \lambda_{J\left(u_{0}\right)}$, then we claim that $u \in \mathcal{N}_{+}$for all $t \in\left[0, T\left(u_{0}\right)\right)$. By contradiction, there exists a $t_{0} \in\left(0, T\left(u_{0}\right)\right)$ such that $u \in \mathcal{N}_{+}$for $0 \leqslant t<t_{0}$ and $u\left(t_{0}\right) \in \mathcal{N}$. Therefore, $u_{t} \neq 0$ for $\Omega \times\left(0, t_{0}\right)$ from (4.11). It follows from (3.5) that $J\left(u\left(t_{0}\right)\right)<J\left(u_{0}\right)$, which implies $u\left(t_{0}\right) \in J^{J\left(u_{0}\right)}$. Furthermore, $u\left(t_{0}\right) \in \mathcal{N}^{J\left(u_{0}\right)}$. By the definition of $\lambda_{J\left(u_{0}\right)}$, we obtain

$$
\left\|u\left(t_{0}\right)\right\|_{L^{2}(\Omega)} \geqslant \lambda_{J\left(u_{0}\right)} .
$$

Noticing that $I(u(t))>0$ for $t \in\left[0, t_{0}\right)$, it follows from (4.11) that

$$
\left\|u\left(t_{0}\right)\right\|_{L^{2}(\Omega)}<\left\|u_{0}\right\|_{L^{2}(\Omega)} \leqslant \lambda_{J\left(u_{0}\right)},
$$

which contradicts (6.4). Therefore, $u \in \mathcal{N}_{+}$for all $t \in\left[0, T\left(u_{0}\right)\right.$ ), then from (4.11) and (3.5), we get $u(t) \in$ $J^{J\left(u_{0}\right)}$ for all $t \in\left[0, T\left(u_{0}\right)\right)$. Lemma 3.8(2) shows that $u(t)$ remains bounded in $X_{0}$ for $t \in\left[0, T\left(u_{0}\right)\right)$, and the boundedness of $\|u\|_{X_{0}}$ is independent of $t$, moreover, $T\left(u_{0}\right)=+\infty, u \in \mathcal{N}_{+} \cap J^{J\left(u_{0}\right)}$ for $0 \leqslant t<\infty$. For any $\omega \in \omega\left(u_{0}\right)$, then

$$
\|\omega\|_{L^{2}(\Omega)}<\lambda_{J\left(u_{0}\right)}, \quad J(\omega)<J\left(u_{0}\right),
$$

by (3.5) and (4.11). Noticing that the definition of $\lambda_{J\left(u_{0}\right)}$, we obtain $\omega\left(u_{0}\right) \cap \mathcal{N}=\emptyset$. Then $\omega\left(u_{0}\right)=\{0\}$, that is, $u_{0} \in \mathcal{G}_{0}$.

(2) If $u_{0} \in \mathcal{N}_{-}$and $\left\|u_{0}\right\|_{L^{2}(\Omega)} \geqslant \Lambda_{J\left(u_{0}\right)}$, then we claim that $u \in \mathcal{N}_{-}$for all $t \in\left[0, T\left(u_{0}\right)\right)$. By contradiction, there exists a $t^{0} \in\left(0, T\left(u_{0}\right)\right)$ such that $u \in \mathcal{N}_{-}$for $0 \leqslant t<t^{0}$ and $u\left(t^{0}\right) \in \mathcal{N}$. Similarly to case (1), we get $J\left(u\left(t^{0}\right)\right)<J\left(u_{0}\right)$, which implies $u\left(t^{0}\right) \in J^{J\left(u_{0}\right)}$. Furthermore, $u\left(t^{0}\right) \in \mathcal{N}^{J\left(u_{0}\right)}$. By the definition of $\Lambda_{J\left(u_{0}\right)}$, we obtain

$$
\left\|u\left(t^{0}\right)\right\|_{L^{2}(\Omega)} \leqslant \Lambda_{J\left(u_{0}\right)} .
$$

Noticing that $I(u(t))<0$ for $t \in\left[0, t^{0}\right)$, it follows from (4.11) that

$$
\left\|u\left(t^{0}\right)\right\|_{L^{2}(\Omega)}>\left\|u_{0}\right\|_{L^{2}(\Omega)} \geqslant \Lambda_{J\left(u_{0}\right)},
$$


which contradicts (6.5). Suppose $T\left(u_{0}\right)=\infty$, then for any $\omega \in \omega\left(u_{0}\right)$,

$$
\|\omega\|_{L^{2}(\Omega)}>\Lambda_{J\left(u_{0}\right)}, \quad J(\omega)<J\left(u_{0}\right),
$$

by (3.5) and (4.11). Noting that the definition of $\Lambda_{J\left(u_{0}\right)}$, we obtain $\omega\left(u_{0}\right) \cap \mathcal{N}=\emptyset$. Then $\omega\left(u_{0}\right)=\{0\}$, which contradicts $\operatorname{dist}\left(0, \mathcal{N}_{-}\right)>0$ in Lemma 3.8(1). Therefore, $\omega\left(u_{0}\right)=\emptyset, T\left(u_{0}\right)<\infty$.

Corollary 6.1. Let conditions $(a)-(d)$ hold, $u_{0} \in X_{0}$. If $d<J\left(u_{0}\right)<\frac{q+1-p}{p(q+1)} \frac{1}{C_{*}^{p}}\left\|u_{0}\right\|_{L^{2}(\Omega)}^{p}$, then $u_{0} \in \mathcal{N}_{-} \cap \mathcal{B}$.

Proof. From condition (c) and the embedding from $X_{0}$ into $L^{2}(\Omega)$, we obtain

$$
\begin{aligned}
J\left(u_{0}\right) & \geqslant \frac{1}{p}\left\|u_{0}\right\|_{X_{0}}^{p}-\frac{1}{q+1} \int_{\Omega} u_{0} f\left(u_{0}\right) \mathrm{d} x \\
& =\frac{q+1-p}{p(q+1)}\left\|u_{0}\right\|_{X_{0}}^{p}+\frac{1}{q+1} I\left(u_{0}\right) \\
& \geqslant \frac{q+1-p}{p(q+1)} \frac{1}{C_{\star}^{p}}\left\|u_{0}\right\|_{L^{2}(\Omega)}^{p}+\frac{1}{q+1} I\left(u_{0}\right) \\
& >J\left(u_{0}\right)+\frac{1}{q+1} I\left(u_{0}\right),
\end{aligned}
$$

which implies $I\left(u_{0}\right)<0$, that is, $u_{0} \in \mathcal{N}_{-}$.

To prove $u_{0} \in \mathcal{B}$, we only need to prove $\left\|u_{0}\right\|_{L^{2}(\Omega)} \geqslant \Lambda_{J\left(u_{0}\right)}$ by Theorem 1.5(2). For any $u \in \mathcal{N}^{J\left(u_{0}\right)}$, the embedding from $X_{0}$ into $L^{2}(\Omega)$ and condition $(c)$ imply that

$$
\begin{aligned}
\frac{q+1-p}{p(q+1)} \frac{1}{C_{\star}^{p}}\|u\|_{L^{2}(\Omega)}^{p} & \leqslant \frac{q+1-p}{p(q+1)}\|u\|_{X_{0}}^{p} \\
& =\frac{q+1-p}{p(q+1)}\|u\|_{X_{0}}^{p}+\frac{1}{q+1} I(u) \\
& =\frac{1}{p}\|u\|_{X_{0}}^{p}-\frac{1}{q+1} \int_{\Omega} u f(u) \mathrm{d} x \\
& \leqslant J(u)<J\left(u_{0}\right) .
\end{aligned}
$$

Therefore, taking the supremum of above inequality over $\mathcal{N}^{J\left(u_{0}\right)}$, we can obtain

$$
\Lambda_{J\left(u_{0}\right)}^{p}<\frac{p(q+1) C_{\star}^{p}}{q+1-p} J\left(u_{0}\right)<\left\|u_{0}\right\|_{L^{2}(\Omega)}^{p},
$$

that is $\left\|u_{0}\right\|_{L^{2}(\Omega)}>\Lambda_{J\left(u_{0}\right)}$. Therefore, $u_{0} \in \mathcal{N}-\cap \mathcal{B}$.

Next, we are concerned with blow-up in finite time for any high initial energy.

Proof of Theorem 1.6. Assume that $M>d$ and $\Omega_{1}, \Omega_{2}$ are two arbitrary disjoint open subdomains of $\Omega$. Denote $Q_{i}=\left(\mathbb{R}^{N} \times \mathbb{R}^{N}\right) \backslash\left(\mathcal{C} \Omega_{i} \times \mathcal{C} \Omega_{i}\right), \mathcal{C} \Omega_{i}=\mathbb{R}^{N} \backslash \Omega_{i}, i=1,2$. Define

$$
X_{0}^{i}=\left\{u \mid u \in L^{p}\left(\Omega_{i}\right), u=0 \text { in } e \Omega_{i}, \frac{|u(x)-u(y)|}{|x-y|^{\frac{N}{p}+s}} \in L^{p}\left(Q_{i}\right)\right\}
$$

for $i=1$, 2. Furthermore, we assume $v \in X_{0}^{1}$ be an arbitrary nonzero function, then we take $\zeta$ large enough such that

$$
J(\zeta v)=\frac{1}{p}\|\zeta v\|_{X_{0}}^{p}-\int_{\Omega} F(\zeta v) \mathrm{d} x \leqslant \frac{1}{p}\|\zeta v\|_{X_{0}}^{p}-B\|\zeta v\|_{L^{q+1}(\Omega)}^{q+1} \leqslant 0,
$$

and

$$
\|\zeta v\|_{L^{2}(\Omega)}^{p}>\frac{p(q+1) C_{\star}^{p}}{q+1-p} M
$$


by Lemma 2.3(2), $q+1>p$. We fix such a number $\zeta>0$ and choose a function $\mu \in X_{0}^{2}$ satisfying $M=J(\mu+\zeta v)=$ $J\left(u_{M}\right)\left(\right.$ Here $\left.u_{M}=\mu+\zeta v\right)$ and it follows that

$$
\left\|u_{M}\right\|_{L^{2}(\Omega)}^{p} \geqslant\|\zeta v\|_{L^{2}(\Omega)}^{p}>\frac{p(q+1) C_{\star}^{p}}{q+1-p} J\left(u_{M}\right) .
$$

By Corollary 6.1, then $u_{M} \in \mathcal{N}_{-} \cap \mathcal{B}$. This completes the proof.

Acknowledgements: The authors would like to thank anonymous reviewers for their inspiring critical comments and thoughtful suggestions to improve the quality of the paper. This research has been partially funded by National Natural Science Foundation of China (No. 11701384, 11671155), China Scholarship Council, NSF of Guangdong (No.2016A030313048) and Natural Science Foundation of SZU (No. 2017057).

\section{References}

[1] B. Abdellaoui, A. Attar, R. Bentifour and I. Peral, On fractional p-Laplacian parabolic problem with general data, Ann. Mat. Pura Appl. 197 (2018), no. 2, 329-356.

[2] C. Bucur and E. Valdinoci, Nonlocal diffusion and applications. Lecture Notes of the Unione Matematica Italiana, 20. Springer, [Cham]; Unione Matematica Italiana, Bologna, 2016. xii+155 pp. ISBN: 978-3-319-287386; 978-3-319-28739-3.

[3] A. Carbotti, S. Dipierro and E. Valdinoci, Local Density of Solutions to Fractional Equations. De Gruyter Studies in Mathematics, 74. De Gruyter, Berlin, 2019. xii+130 pp. ISBN: 978-3-11-066435-5.

[4] G.Y. Chen, J.C. Wei and Y.F. Zhou, Finite time blow-up for the fractional critical heat equation in $\mathbb{R}^{n}$, Nonlinear Analysis (2019), doi: https://doi.org/10.1016/j.na.2019.01.010.

[5] E. Di Nezza, G. Palatucci and E. Valdinoci, Hitchhiker's guide to the fractional Sobolev spaces, Bull. Sci. Math. 136 (2012), no. 5, 521-573.

[6] S. Dipierro, M. Medina and E. Valdinoci, Fractional elliptic problems with critical growth in the whole of $\mathbb{R}^{n}$. Appunti. Scuola Normale Superiore di Pisa (Nuova Serie) [Lecture Notes. Scuola Normale Superiore di Pisa (New Series)], 15. Edizioni della Normale, Pisa, 2017. viii+152 pp. ISBN: 978-88-7642-600-1; 978-88-7642-601-8.

[7] Z.H. Dong and J. Zhou, Global existence and finite time blow-up for a class of thin-film equation, Z. Angew. Math. Phys. 68 (2017), no. 4, $17 \mathrm{pp}$.

[8] C.G. Gal and M. Warma, On some degenerate non-local parabolic equation associated with the fractional $p$-Laplacian, Dyn. Partial Differ. Equ. 14 (2017), no. 1, 47-77.

[9] J. Giacomoni and S. Tiwari, Existence and global behavior of solutions to fractional $p$-laplacian parabolic problems, Electron. J. Differential Equations 2018 (2018), no. 44, 1-20.

[10] G. Gilboa and S. Osher, Nonlocal operators with applications to image processing, Multiscale Model. Simul. 7 (2008), no. 3, 1005-1028.

[11] Y.Z. Han, A class of fourth-order parabolic equation with arbitrary initial energy, Nonlinear Anal. Real World Appl. 43 (2018), 451-466.

[12] R. Hynd and E. Lindgren, Hölder estimates and large time behavior for a nonlocal doubly nonlinear evolution, Anal. PDE 9 (2016), no. 6, 1447-1482.

[13] V. K. Kalantarov and O. A. Ladyzhenskaya, The occurrence of collapse for quasilinear equations of parabolic and hyperbolic types, Zap. Nauchn. Sem. LOMI 69 (1977), 77-102.

[14] N. S. Landkof, Foundations of Modern Potential Theory, vol. 180 of Grundlehren Math. Wiss., Springer, Berlin, 1972.

[15] H.A. Levine, Some nonexistence and instability theorems for solutions of formally parabolic equations of the form $P u_{t}=$ $-A u+F(u)$, Arch. Rational Mech. Anal. 51 (1973), 371-386.

[16] M.L. Liao and W.J. Gao, Blow-up phenomena for a nonlocal $p$-Laplace equation with Neumann boundary conditions, Arch. Math. (Basel) 108 (2017), no. 3, 313-324.

[17] Y.C. Liu, On potential wells and vacuum isolating of solutions for semilinear wave equations, J. Differential Equations 192 (2003), no. 1, 155-169.

[18] Y.C. Liu and J.S. Zhao, On potential wells and applications to semilinear hyperbolic equations and parabolic equations, Nonlinear Anal. 64 (2006), no. 12, 2665-2687.

[19] J.M. Mazón, J.D. Rossi and J. Toledo, Fractional p-Laplacian evolution equations, J. Math. Pures Appl. 105 (2016), no. 6, 810-844.

[20] G. Molica Bisci, V.D. Radulescu and R. Servadei, Variational methods for nonlocal fractional problems. With a foreword by Jean Mawhin. Encyclopedia of Mathematics and its Applications, 162. Cambridge University Press, Cambridge, 2016. xvi+383 pp. ISBN: 978-1-107-111943.

[21] M. Musso, Y. Sire, J.C. Wei, Y.Q. Zheng and Y.F. Zhou, Infinite time blow-up for the fractional heat equation with critical exponent, Mathematische Annalen 375 (2019), no. 1-2, 361-424. 
[22] N. Pan, B.L. Zhang and J. Cao, Degenerate Kirchhoff-type diffusion problems involving the fractional $p$-Laplacian, Nonlinear Anal. Real World Appl. 37 (2017) 56-70.

[23] L.E. Payne and D.H. Sattinger, Saddle points and instability of nonlinear hyperbolic equations, Israel J. Math. 22 (1975), no. 3-4, 273-303.

[24] D. Puhst, On the evolutionary fractional p-Laplacian, Appl. Math. Res. Express. AMRX 2015 (2015), no. 2, $253-273$.

[25] D.H. Sattinger, On global solution of nonlinear hyperbolic equations, Arch. Rational Mech. Anal. 30 (1968) 148-172.

[26] R. Servadei and E. Valdinoci, Mountain pass solutions for non-local elliptic operators, J. Math. Anal. Appl. 389 (2012), no. 2, 887-898.

[27] R. Servadei and E. Valdinoci, Variational methods for non-local operators of elliptic type, Discrete Contin. Dyn. Syst. 33 (2013), no. 5, 2105-2137.

[28] L. E. Silvestre, Regularity of the obstacle problem for a fractional power of the Laplace operator. Thesis (Ph.D.)-The University of Texas at Austin. 2005. 95 pp. ISBN: 978-0542-25310-2.

[29] J. Simon, Compact sets in the space $L^{p}(0, T ; B)$, Ann. Mat. Pura Appl. 146 (1987), no. 4, 65-96.

[30] E. Stein, Singular Integrals and Differentiability Properties of Functions, Princeton University Press, Princeton, New Jersey, 1970.

[31] J.L. Vázquez, Nonlinear diffusion with fractional Laplacian operators, in: H.Holden, K. H. Karlsen (eds.), Nonlinear Partial Differential Equations, The Abel Symposium 2010, Springer, Berlin, 2012, pp. 271-298.

[32] J.L. Vázquez, The Dirichlet problem for the fractional $p$-Laplacian evolution equation, J. Differential Equations 260 (2016), no. 7, 6038-6056.

[33] M.Q. Xiang, B.L. Zhang and M. Ferrara, Existence of solutions for Kirchhoff type problem involving the non-local fractional p-Laplacian, J. Math. Anal. Appl. 424 (2015), no. 2, 1021-1041.

[34] R.Z. Xu and J. Su, Global existence and finite time blow-up for a class of semilinear pseudo-parabolic equations, J. Funct. Anal. 264 (2013), no. 12, 2732-2763.

[35] G.Y. Xu and J. Zhou, Global existence and finite time blow-up of the solution for a thin-film equation with high initial energy, J. Math. Anal. Appl. 458 (2018), no. 1, 521-535. 